\title{
Fixed Point Theorems for Non-compatible, Discontinuous Hybrid Pairs of Mappings on 2-Metric Spaces by Using Implicit Relation
}

\author{
R. D. Daheriya ${ }^{1 *}$, Motiram Likhitker ${ }^{1}$, Manoj Ughade ${ }^{2}$ \\ ${ }^{1}$ Department of Mathematics, Government J. H. P.G. College, Betul, P.O. Box 460225, India \\ ${ }^{2}$ Department of Mathematics, SRK University, Bhopal, P.O. Box 462026, India \\ "Correspondingauthor, e-mail: mrlikhitker950@gmail.com
}

\begin{abstract}
In this article, we prove a number of common fixed point theorems for hybrid pairs of mappings satisfying an implicit contraction relation by using weak commutativity of type (KB) in the setting of a 2-metric space. Also, we present an example to illustrate the effectiveness of our results.
\end{abstract}

Keywords: Coincidence point, Common fixed point, D-maps, Weak commutativity of type (KB), implicit contraction relation.

2010 Mathematics Subject Classification: 47H10, 54H25.

\section{DEFINITION AND NotaTION}

The concept of a 2-metric space is a natural generalization of a metric space. It has been introduced by Gähler ([3]-[5]) and extensively studied by some mathematicians such as Gähler ([3]-[5]), White [18], Iséki [6]. Moreover, a number of authors ([1], [10], [13], [17]) have studied the contractive, nonexpansive and contraction type mapping in 2-metric spaces. On the other hand, Jungck [7] studied the common fixed points of commuting maps. Then Sessa [16] generalized the commuting maps by introducing the notion of weakly commuting and proved a common fixed point theorem for weakly commuting maps. Jungck [8] further made a generalization of weakly commuting maps by introducing the notion of compatible mappings. Moreover, Jungck and Rhoades [9] introduced the notion of coincidentally commuting or weakly compatible mappings. Several authors used these concepts to prove some common fixed point theorems on usual metric, as well as on different kinds of generalized metric spaces ([1], [2], [11], [15]). In this paper, the existence and approximation of a unique common fixed point of two families of weakly compatible self maps on a 2-metric space are proved. Pant ([20]-[23]) initiated the study of non-compatible maps and introduced pointwise R-weak commutativity of mappings in [20]. He also showed that point wise R-weak commutativity is a necessary, hence minimal, condition for the existence of a common fixed point of contractive type maps [21]. Pathak et al. [24] introduced the concept of R-weakly commuting maps of type (A), and showed that they are not compatible. Kubiaczyk and Deshpande [19] extended the concept of Rweakly commutativity of type (A) for single valued mappings to set valued mappings and introduced weak commutativity of type $(\mathrm{KB})$ which is a weaker condition than $\delta$-compatibility. In fact, $\delta$ compatibility maps are weakly commuting of type $(\mathrm{KB})$ but converse is not true. For example we can see [19], [25 and [26]. Recently, Sharma and Deshpande [25] proved a common fixed point theorem for two pairs of hybrid mappings by using weak commutativity of type (KB) on a non-complete metric space without assuming continuity of any mapping.

In this paper, we present a number of common fixed point theorems for hybrid pairs of mappings satisfying an implicit contraction relation in the setting of a 2-metric space by using weak commuting of type (KB). In Section 2.4, we give an example to illustrate the effectiveness of our results.

\section{Preliminaries}

Throughout this paper, we will adopt the following notations: $\mathbb{N}$ is the set of all natural numbers, $\mathbb{R}^{+}$ is the set of all non-negative real numbers. For mappings $\mathrm{I}: \mathrm{X} \rightarrow \mathrm{X}$ and $\mathrm{F}: \mathrm{X} \rightarrow \mathcal{B}(\mathrm{X})$, we denote 


$$
\begin{aligned}
\mathcal{F}[I] & =\{\mathrm{X} \in \mathrm{X}: \mathrm{X}=\mathrm{IX}, \text { i. e. } \mathrm{x} \text { is a fixed point of } \mathrm{I}\}, \\
\mathcal{F}[\mathrm{F}] & =\{\mathrm{x} \in \mathrm{X}:\{\mathrm{X}\}=\mathrm{FX} \text {, i. e. } \mathrm{x} \text { is a fixed point of } \mathrm{F}\}, \\
\mathcal{C}[\mathrm{I}, \mathrm{F}] & =\{\mathrm{X} \in \mathrm{X}:\{\mathrm{IX}\}=\mathrm{FX} \text {, i. e. } \mathrm{X} \text { is a coincidence point of } \mathrm{I} \text { and } \mathrm{F}\}
\end{aligned}
$$

Let $\mathcal{B}(\mathrm{X})$ be the class of all nonempty bounded subsets of $\mathrm{X}$. For all $\mathrm{A}, \mathrm{B}, \mathrm{C} \in \mathcal{B}(\mathrm{X})$, let $\delta(\mathrm{A}, \mathrm{B}, \mathrm{C})$ and $D(A, B, C)$ be the functions defined by

$$
\begin{aligned}
& \delta(A, B, C)=\sup \{d(a, b, c): a \in A, b \in B, c \in C\} \\
& \mathcal{D}(A, B, C)=\inf \{d(a, b, c): a \in A, b \in B, c \in C\}
\end{aligned}
$$

If $\mathrm{A}$ consists of a single point a, we write $\delta(\mathrm{A}, \mathrm{B}, \mathrm{C})=\delta(\mathrm{a}, \mathrm{B}, \mathrm{C})$. If $\mathrm{B}$ and $\mathrm{C}$ also consist of single points $\mathrm{b}$ and $\mathrm{c}$, respectively, we write

$$
\delta(\mathrm{A}, \mathrm{B}, \mathrm{C})=\mathcal{D}(\mathrm{A}, \mathrm{B}, \mathrm{C})=\delta(\mathrm{a}, \mathrm{b}, \mathrm{c})
$$

It follows immediately from the definition that: for all $A, B, C, E \in B(X)$,

$$
\begin{aligned}
& \delta(A, B, C)=\delta(A, C, B)=\delta(C, B, A)=\delta(C, A, B) \\
&=\delta(B, C, A)=\delta(B, A, C) \geq 0, \\
& \delta(A, B, C) \leq \delta(A, B, E)+\delta(A, E, C)+\delta(E, B, C)
\end{aligned}
$$

If at least two of $\mathrm{A}, \mathrm{B}$ and $\mathrm{C}$ are singleton, then $\delta(\mathrm{A}, \mathrm{B}, \mathrm{C})=0$.

In order to study these theorems, we recall the definition of a 2-metric space which is given by Gähler as follows:

Definition 2.1 (see [3]) Let $\mathrm{X}$ be a nonempty set. A real valued function $\mathrm{d}$ on $\mathrm{X}^{3}$ is said to a 2-metric if,

[M1] To each pair of distinct points $\mathrm{x}, \mathrm{y}$ in $\mathrm{X}$, there exists a point $\mathrm{z} \in \mathrm{X}$ such that $d(x, y, z) \neq 0$,

[M1] $d(x, y, z)=0$ when at least two of $x, y, z$ are equal,

[M2] $d(x, y, z)=d(x, z, y)=d(y, z, x)$,

[M3] $\quad D(x, y, z) \leq d(x, y, u)+d(x, u, z)+d(u, y, z)$ for all $x, y, z, u \in X$.

The function $\mathrm{d}$ is called a 2-metric on the set $\mathrm{X}$ whereas the pair $(\mathrm{X}, \mathrm{d})$ stands for 2-metric space. Geometrically a 2-metric $d(x, y, z)$ represents the area of a triangle with vertices $x, y$ and $z$.

If has been know since Gahler [9] that a 2-metric $d$ is a non-negative continuous function in any of its three arguments. A 2-metric d is said to be continuous, if it is continuous in all of its arguments. Throughout this paper $\mathrm{d}$ stands for a continuous 2-metric.

Definition 2.2 (see [14]) A sequence $\left\{x_{n}\right\}$ in a 2-metric space $(X, d)$ is said to be convergent to a point $x \in X$, if $\lim _{n \rightarrow \infty} d\left(x_{n}, x, z\right)=0$ for all $z \in X$.

Definitions 2.3 (see [14]) A sequence $\left\{x_{n}\right\}$ in a 2-metric space $(X, d)$ is said to be Cauchy sequence if $\lim _{n \rightarrow \infty} d\left(x_{n}, x_{m}, z\right)=0$ for all $z \in X$.

Definitions 2.4 (see [14]) A 2-metric space (X, d) is said to be complete if every Cauchy sequence in $\mathrm{X}$ is convergent.

Remark 2.1 We note that, in a metric space a convergent sequence is a Cauchy sequence and in a 2metric space a convergent sequence need not be a Cauchy sequence, but every convergent sequence is a Cauchy sequence when the 2-metric $\mathrm{d}$ is continuous on $\mathrm{X}$ [12]. 
Point Theorems for Non-compatible, Discontinuous Hybrid Pairs of Mappings on 2-Metric Spaces by Using Implicit Relation

Definition 2.5 (see [1]) A sequence $\left\{A_{n}\right\}$ of subsets of a 2-metric space $(X, d)$ is said to be convergent to a subset $\mathrm{A}$ of $\mathrm{X}$ if:

1. given $a \in A$, there is a sequence $\left\{a_{n}\right\}$ in $X$ such that $a_{n} \in A_{n}$ for $n=1,2,3 \ldots$ and $\lim _{n \rightarrow \infty} d\left(a_{n}, a, c\right) \forall c \in X$.

2. given " $\varepsilon>0$, there exists a positive integer $N$ such that $A_{n} \subseteq A_{\epsilon}$ for $n>N$ where $A_{\epsilon}$ is the union of all open spheres with centers in A and radius".

Definition 2.6 (see [1]) The mappings $F: X \rightarrow \mathcal{B}(X)$ and $I: X \rightarrow X$ are said to be weakly commuting on $\mathrm{X}$ if IFX $\in \mathcal{B}(\mathrm{X})$ and for all $\mathrm{C}$ in $\mathcal{B}(\mathrm{X})$,

$$
\delta(\mathrm{FIx}, \mathrm{IFx}, \mathrm{C}) \leq \max \{\delta(\mathrm{Ix}, \mathrm{Fx}, \mathrm{C}), \delta(\mathrm{IFx}, \mathrm{IFx}, \mathrm{C})\} .
$$

Note that if $\mathrm{F}$ is a single valued mapping, then the set IFx consists of a single point. Therefore, $\delta(\operatorname{IFx}, \mathrm{IFx}, \mathrm{C})=\mathcal{D}(\mathrm{IFx}, \mathrm{IFx}, \mathrm{C})=0$ for all $\mathrm{C}$ in $\mathcal{B}(\mathrm{X})$ and the above inequality reduces to the condition given by Khan [27], that is $\mathcal{D}(\mathrm{FIx}, \mathrm{IFx}, \mathrm{C}) \leq \mathcal{D}(\mathrm{Ix}, \mathrm{Fx}, \mathrm{C})$.

Definition 2.7 (see [1]) The mappings $\mathrm{F}: \mathrm{X} \rightarrow \mathcal{B}(\mathrm{X})$ and $\mathrm{I}: \mathrm{X} \rightarrow \mathrm{X}$ are said to be compatible if $\lim _{n \rightarrow \infty} \delta\left(F_{x_{n}}, I F x_{n}, C\right)=0$ for all $C$ in $\mathcal{B}(X)$, whenever $\left\{x_{n}\right\}$ is a sequence in $X$ such that $\lim _{n \rightarrow \infty} I x_{n}=t$ and $\lim _{n \rightarrow \infty} F_{n}=\{t\}$ for some $t \in X$.

Definition 2.8 (see [29]) The mappings $\mathrm{F}: \mathrm{X} \rightarrow \mathcal{B}(\mathrm{X})$ and $\mathrm{I}: \mathrm{X} \rightarrow \mathrm{X}$ are said to be D-maps if and only if there exists a sequence $\left\{\mathrm{x}_{\mathrm{n}}\right\}$ in $\mathrm{X}$ such that $\mathrm{IFx} \in \mathcal{B}(\mathrm{X}), \mathrm{Fx}_{\mathrm{n}} \rightarrow\{\mathrm{t}\}$ and $\mathrm{fx}_{\mathrm{n}} \rightarrow \mathrm{t}$ for some $\mathrm{t}$ in $\mathrm{X}$.

Definition 2.9 (see [1]) The mappings $\mathrm{F}: \mathrm{X} \rightarrow \mathcal{B}(\mathrm{X})$ and I $: \mathrm{X} \rightarrow \mathrm{X}$ are said to be $\delta$-compatible if $\lim _{n \rightarrow \infty} \delta\left(F x_{n}, I F x_{n}, C\right)=0$ for all $C$ in $\mathcal{B}(X)$, whenever $\left\{x_{n}\right\}$ is a sequence in $X$ such that IFx $\in$ $\mathcal{B}(\mathrm{X}), \mathrm{Fx}_{\mathrm{n}} \rightarrow\{\mathrm{t}\}$ and $\mathrm{Ix}_{\mathrm{n}} \rightarrow \mathrm{t}$ for some $\mathrm{t}$ in $\mathrm{X}$.

Example 2.1 Define $d$ on $[0,10) \times[0,10) \times[0,10)$ by $d(x, y, z)=\min \{\varrho(x, y), \varrho(y, z), \varrho(z, x)\}$, where $\varrho$ is a usual metric on $[0,10)$. Then it is easy to see that $([0,10), d)$ is a 2 -metric space. Define mappings I, J: $[0,10) \rightarrow[0,10)$ and $\mathrm{F}, \mathrm{G}:[0,10) \rightarrow \mathcal{B}([0,10))$ by

$$
\begin{aligned}
& I x=\left\{\begin{array}{ll}
x, & x \in[0,5], \\
\frac{2 x+4}{7}, & x \in(5,10),
\end{array} \quad J x=\left\{\begin{array}{lr}
2 x, & x \in[0,5], \\
\frac{x-1}{2}, & x \in(5,10),
\end{array}\right.\right. \\
& F x=\left\{\begin{array}{ll}
{[0, x],} & x \in[0,5], \\
{\left[1, \frac{3 x+5}{10}\right],} & x \in(5,10),
\end{array} \quad G x=\left\{\begin{array}{l}
{\left[0, \frac{x}{2}\right], \quad x \in[0,5],} \\
{\left[1, \frac{x+5}{5}\right], x \in(5,10),}
\end{array}\right.\right.
\end{aligned}
$$

Define a sequence $\left\{x_{n}\right\}$ by $x_{n}=5+\frac{1}{n}$ in $[0,10)$. Obviously, $\lim _{n \rightarrow \infty} x_{n}=5 \in[0,10)$ and then

$$
\begin{array}{ll}
\lim _{n \rightarrow \infty} \operatorname{Ix}_{n}=\lim _{n \rightarrow \infty} \frac{2 x_{n}+4}{7}=2, & \lim _{n \rightarrow \infty} J x_{n}=\lim _{n \rightarrow \infty} \frac{x_{n}-1}{2}=2, \\
\lim _{n \rightarrow \infty} F=\lim _{n \rightarrow \infty}\left[1, \frac{3 x_{n}+5}{10}\right]=[1,2], & \lim _{n \rightarrow \infty} \mathrm{Gx}_{n}=\lim _{n \rightarrow \infty}\left[1, \frac{x_{n}+5}{5}\right]=[1,2] .
\end{array}
$$

Clearly, $2 \in[1,2]$. Therefore, $I$ and $F$ are D-maps and $J$ and $G$ are D-maps. Notice that $\lim _{n \rightarrow \infty} \delta\left(\mathrm{FIx}_{\mathrm{n}}, \mathrm{IFx}_{\mathrm{n}}, \mathrm{C}\right) \neq 0$ and $\lim _{\mathrm{n} \rightarrow \infty} \delta\left(\mathrm{GJx}_{\mathrm{n}}, J G x_{\mathrm{n}}, \mathrm{C}\right) \neq 0$. Therefore the hybrid pairs $\{\mathrm{F}, \mathrm{I}\}$ and $\{\mathrm{G}, \mathrm{J}\}$ are not $\delta$-compatible.

Definition 2.10 (see [8]) The mappings $\mathrm{F}: \mathrm{X} \rightarrow \mathcal{B}(\mathrm{X})$ and $\mathrm{I}: \mathrm{X} \rightarrow \mathrm{X}$ are said to be weakly compatible if they commute at a coincidence point $\mathrm{u}$ in $\mathrm{X}$ such that $\mathrm{Fu}=\{\mathrm{Iu}\}$ we have $\mathrm{FIu}=\mathrm{IFu}$.

Note that the equation $\mathrm{Fu}=\{\mathrm{Iu}\}$ implies that $\mathrm{Fu}$ is a singleton. It can be easily shown that any $\delta$ compatible pair $\{\mathrm{F}, \mathrm{I}\}$ is weakly compatible but the converse is false. 


\section{R.D. Daheriya et al.}

Definition 2.11 (see [19]) The mappings $F: X \rightarrow \mathcal{B}(X)$ and $I: X \rightarrow X$ are said to be weakly commuting of type $(\mathrm{KB})$ at $\mathrm{x}$ if there exists some positive real number $\mathrm{R}$ such that for all $\mathrm{C}$ in $\mathcal{B}(\mathrm{X})$, $\delta(\mathrm{IIx}, \mathrm{FIx}, \mathrm{C}) \leq \mathrm{R} \delta(\mathrm{Ix}, \mathrm{Fx}, \mathrm{C})$.

Here $\mathrm{F}$ and I are weakly commuting of type $(\mathrm{KB})$ on $\mathrm{X}$ if the above inequality holds for all $\mathrm{x} \in \mathrm{X}$. Every $\delta$-compatible pair of hybrid maps is weakly commuting of type (KB) but the converse is not necessarily true. For example we can see [19], [25] and [26].

Lemma 2.1 (see [1]) If $\left\{A_{n}\right\}$ and $\left\{B_{n}\right\}$ are sequences in $\mathcal{B}(X)$ converging to $A$ and $B$ in $\mathcal{B}(X)$ respectively, then the sequence $\left\{\delta\left(A_{n}, B_{n}, C\right)\right\}$ converges to $\delta(A, B, C)$.

A class of implicit relation: $\Phi$ denotes a family of mappings such that $\varphi \in \Phi, \varphi:\left(\mathbb{R}^{+}\right)^{5} \rightarrow \mathbb{R}^{+}$and $\varphi$ is continuous and increasing in each coordinate variable. Also $\gamma(\mathrm{t})=\varphi\left(\mathrm{t}, \mathrm{t}, \mathrm{a}_{1} \mathrm{t}, \mathrm{a}_{2} \mathrm{t}, \mathrm{t}\right)<t$ for every $t \in \mathbb{R}^{+}$, where $a_{1}+a_{2}=3$.

Example 2.2 Let $\varphi:\left(\mathbb{R}^{+}\right)^{5} \rightarrow \mathbb{R}^{+}$be defined by $\varphi\left(t_{1}+t_{2}+t_{3}+t_{4}+t_{5}\right)=\frac{1}{7}\left(t_{1}+t_{2}+t_{3}+t_{4}+\right.$ t5.Obviously, the function $\varphi$ is continuous and increasing in each coordinate variable. Also $\gamma \mathrm{t}=\varphi \mathrm{t}, \mathrm{t}$, $\mathrm{a} 1 \mathrm{t}, \mathrm{a} 2 \mathrm{t}, \mathrm{t}=\mathrm{t} 73+\mathrm{a} 1+\mathrm{a} 2<t$, where $\mathrm{a} 1+\mathrm{a} 2=3$. Thus, the function $\varphi \in \Phi$.

The following lemma is the key in proving our result.

Lemma 2.2 (see [28]) For every $t>0, \gamma(t)<t$ if and only if $\lim _{n \rightarrow \infty} \gamma^{n}(t)=0$, where $\gamma^{n}$ denotes the composition of $\gamma$ with itself $n$ times.

\section{Main Result}

The following proposition notes that in the following specific setting the common fixed point of the involved four mappings is always unique provided it exists.

Proposition 3.1 Let $(\mathrm{X}, \mathrm{d})$ be a metric space. Let $\mathrm{I}, \mathrm{J}$ be mappings of $\mathrm{X}$ into itself and $\mathrm{F}, \mathrm{G}$ of $\mathrm{X}$ into $\mathcal{B}(\mathrm{X})$ satisfying the condition:

$$
\delta(\mathrm{Fx}, \mathrm{Gy}, \mathrm{C}) \leq \varphi(\delta(\mathrm{Ix}, \mathrm{Jy}, \mathrm{C}), \delta(\mathrm{Ix}, \mathrm{Fx}, \mathrm{C}), \delta(\mathrm{Jy}, \mathrm{Gy}, \mathrm{C}), \mathcal{D}(\mathrm{Ix}, \mathrm{Gy}, \mathrm{C}), \mathcal{D}(\mathrm{Jy}, \mathrm{Fx}, \mathrm{C}))
$$

for all $\mathrm{x}, \mathrm{y} \in \mathrm{X}$ and $\mathrm{C} \in \mathcal{B}(\mathrm{X})$, where $\varphi \in \Phi$. Then $(\mathcal{F}[\mathrm{I}] \cap \mathcal{F}[\mathrm{J}] \cap \mathcal{F}[\mathrm{F}] \cap \mathcal{F}[\mathrm{G}])$ is a singleton set, that is, there exists a point $\mathrm{z} \in \mathrm{X}$ such that $(\mathcal{F}[\mathrm{I}] \cap \mathcal{F}[\mathrm{J}] \cap \mathcal{F}[\mathrm{F}] \cap \mathcal{F}[\mathrm{G}])=\{\mathrm{z}\}$.

Proof Suppose, to the contrary, that the set $(\mathcal{F}[\mathrm{I}] \cap \mathcal{F}[\mathrm{J}] \cap \mathcal{F}[\mathrm{F}] \cap \mathcal{F}[\mathrm{G}])$ is not singleton. Then there exist two points $\mathrm{z}$ and $\mathrm{w}, \mathrm{z} \neq \mathrm{w}$ in $\mathrm{X}$ such that $\{\mathrm{z}\}=\{\mathrm{Iz}\}=\{\mathrm{Jz}\}=\mathrm{Fz}=\mathrm{Gz}$ and $\{\mathrm{w}\}=\{\mathrm{Iw}\}=$ $\{J w\}=F w=G w$. Since $\varphi$ is an increasing function, by (3.1), we have;

$$
\begin{aligned}
\delta(\mathrm{z}, \mathrm{w}, \mathrm{C}) & \leq \delta(\mathrm{Fz}, \mathrm{Gw}, \mathrm{C}) \\
& \leq \varphi(\delta(\mathrm{Iz}, \mathrm{Jw}, \mathrm{C}), \delta(\mathrm{Iz}, \mathrm{Fz}, \mathrm{C}), \delta(\mathrm{Jw}, \mathrm{Gw}, \mathrm{C}), \mathcal{D}(\mathrm{Iz}, \mathrm{Gw}, \mathrm{C}), \mathcal{D}(\mathrm{Jw}, \mathrm{Fz}, \mathrm{C})) \\
& \leq \varphi(\delta(\mathrm{z}, \mathrm{w}, \mathrm{C}), 0,0, \delta(\mathrm{z}, \mathrm{w}, \mathrm{C}), \delta(\mathrm{w}, \mathrm{z}, \mathrm{C})) \\
& \leq \varphi(\delta(\mathrm{z}, \mathrm{w}, \mathrm{C}), \delta(\mathrm{z}, \mathrm{w}, \mathrm{C}), 2 \delta(\mathrm{z}, \mathrm{w}, \mathrm{C}), \delta(\mathrm{z}, \mathrm{w}, \mathrm{C}), \delta(\mathrm{z}, \mathrm{w}, \mathrm{C})) \\
& \leq \varphi(\delta(\mathrm{z}, \mathrm{w}, \mathrm{C}))<\delta(\mathrm{z}, w, C)
\end{aligned}
$$

Here we reach a contradiction. Thus, our supposition that the set $(\mathcal{F}[\mathrm{I}] \cap \mathcal{F}[\mathrm{J}] \cap \mathcal{F}[\mathrm{F}] \cap \mathcal{F}[\mathrm{G}])$ is not singleton was wrong. Hence $(\mathcal{F}[\mathrm{I}] \cap \mathcal{F}[J] \cap \mathcal{F}[\mathrm{F}] \cap \mathcal{F}[\mathrm{G}])$ is a singleton set.

Theorem 3.2 Let (X, d) be a metric space. Let $\mathrm{I}$, J be mappings of $\mathrm{X}$ into itself and $\mathrm{F}, \mathrm{G}$ of $\mathrm{X}$ into $\mathcal{B}(\mathrm{X})$ satisfying the condition (3.1). Then $(\mathcal{F}[\mathrm{I}] \cap \mathcal{F}[\mathrm{J}]) \cap \mathcal{F}[\mathrm{F}]=(\mathcal{F}[\mathrm{I}] \cap \mathcal{F}[\mathrm{J}]) \cap \mathcal{F}[\mathrm{G}]$

Proof Let $\mathrm{z} \in(\mathcal{F}[\mathrm{I}] \cap \mathcal{F}[\mathrm{J}]) \cap \mathcal{F}[\mathrm{F}]$. We will prove that $\mathrm{z} \in(\mathcal{F}[\mathrm{I}] \cap \mathcal{F}[\mathrm{J}]) \cap \mathcal{F}[\mathrm{G}]$. Suppose, to the contrary, that $\mathrm{z} \notin(\mathcal{F}[\mathrm{I}] \cap \mathcal{F}[\mathrm{J}]) \cap \mathcal{F}[\mathrm{G}]$. Then using (3.1), we have; for all $\mathrm{C} \in \mathcal{B}(\mathrm{X})$ 


$$
\begin{aligned}
\delta(\mathrm{z}, \mathrm{Gz}, \mathrm{C}) & =\delta(\mathrm{Fz}, \mathrm{Gz}, \mathrm{C}) \\
& \leq \varphi(\delta(\mathrm{Iz}, \mathrm{Jz}, \mathrm{C}), \delta(\mathrm{Iz}, \mathrm{Fz}, \mathrm{C}), \delta(\mathrm{Jz}, \mathrm{Gz}, \mathrm{C}), \mathcal{D}(\mathrm{Iz}, \mathrm{Gz}, \mathrm{C}), \mathcal{D}(\mathrm{Jz}, \mathrm{Fz}, \mathrm{C})) \\
& =\varphi(\delta(\mathrm{z}, \mathrm{z}, \mathrm{C}), \delta(\mathrm{z}, \mathrm{z}, \mathrm{C}), \delta(\mathrm{z}, \mathrm{Gz}, \mathrm{C}), \delta(\mathrm{z}, \mathrm{Gz}, \mathrm{C}), \delta(\mathrm{z}, \mathrm{z}, \mathrm{C})) \\
& \leq \varphi(0,0, \delta(\mathrm{z}, \mathrm{Gz}, \mathrm{C}), \delta(\mathrm{z}, \mathrm{Gz}, \mathrm{C}), 0) \\
& \leq \varphi(\delta(\mathrm{z}, \mathrm{Gz}, \mathrm{C}), \delta(\mathrm{z}, \mathrm{Gz}, \mathrm{C}), 2 \delta(\mathrm{z}, \mathrm{Gz}, \mathrm{C}), \delta(\mathrm{z}, \mathrm{Gz}, \mathrm{C}), \delta(\mathrm{z}, \mathrm{Gz}, \mathrm{C})) \\
& \leq \gamma(\delta(\mathrm{z}, \mathrm{Gz}, \mathrm{C}))<\delta(z, G z, C)
\end{aligned}
$$

we reach a contradiction. Thus, our supposition that $\mathrm{z} \notin(\mathcal{F}[\mathrm{I}] \cap \mathcal{F}[\mathrm{J}]) \cap \mathcal{F}[\mathrm{G}]$ was wrong. Hence $\mathrm{z} \in(\mathcal{F}[\mathrm{I}] \cap \mathcal{F}[\mathrm{J}]) \cap \mathcal{F}[\mathrm{G}]$ and then $(\mathcal{F}[\mathrm{I}] \cap \mathcal{F}[\mathrm{J}]) \cap \mathcal{F}[\mathrm{F}] \subseteq(\mathcal{F}[\mathrm{I}] \cap \mathcal{F}[\mathrm{J}]) \cap \mathcal{F}[\mathrm{G}]$. Similarly, one can show that $(\mathcal{F}[\mathrm{I}] \cap \mathcal{F}[\mathrm{J}]) \cap \mathcal{F}[\mathrm{G}] \subseteq(\mathcal{F}[\mathrm{I}] \cap \mathcal{F}[\mathrm{J}]) \cap \mathcal{F}[\mathrm{F}]$. Thus, it follows that $(\mathcal{F}[\mathrm{I}] \cap \mathcal{F}[\mathrm{J}]) \cap$ $\mathcal{F}[\mathrm{F}]=(\mathcal{F}[\mathrm{I}] \cap \mathcal{F}[\mathrm{J}]) \cap \mathcal{F}[\mathrm{G}]$.

Let $(\mathrm{X}, \mathrm{d})$ be a metric space. Let $\mathrm{I}, \mathrm{J}$ be mappings of $\mathrm{X}$ into itself and $\mathrm{F}, \mathrm{G}$ of $\mathrm{X}$ into $\mathcal{B}(\mathrm{X})$ satisfying condition:

$$
\mathrm{U} F(\mathrm{X}) \subseteq \mathrm{J}(\mathrm{X}) \quad \text { and } \quad \mathrm{U} \mathrm{G}(\mathrm{X}) \subseteq \mathrm{I}(\mathrm{X})
$$

Let $\mathrm{x}_{0}$ be an arbitrary point in $\mathrm{X}$. By (3.2), we choose a point $\mathrm{x}_{1}$ in $\mathrm{X}$ such that $\mathrm{J} \mathrm{x}_{1} \in \mathrm{Fx}_{0}=\mathrm{Z}_{0}$ and for this point $\mathrm{x}_{1}$ there exist a point $\mathrm{x}_{1}$ in $\mathrm{X}$ such that $\mathrm{Ix}_{2} \in \mathrm{Gx}_{1}=\mathrm{Z}_{1}$ and so on. Continuing in this manner, we can define a sequence $\left\{\mathrm{x}_{\mathrm{n}}\right\}$ as follows:

$$
\mathrm{Jx}_{2 \mathrm{n}+1} \in \mathrm{Fx}_{2 \mathrm{n}}=\mathrm{Z}_{2 \mathrm{n}}, \mathrm{Ix}_{2 \mathrm{n}+2} \in \mathrm{Gx}_{2 \mathrm{n}+1}=\mathrm{Z}_{2 \mathrm{n}+1}, \forall \mathrm{n} \in \mathbb{N} \cup\{0\}
$$

For simplicity, we set:

$$
\mathrm{V}_{\mathrm{n}}(\mathrm{C})=\delta\left(\mathrm{Z}_{\mathrm{n}}, \mathrm{Z}_{\mathrm{n}+1}, \mathrm{C}\right), \quad \mathrm{n} \in \mathbb{N} \cup\{0\}
$$

In the following we introduce some auxiliary lemmas are useful in the sequel.

Lemma 3.1 Let $(\mathrm{X}, \mathrm{d})$ be a metric space. Let $\mathrm{I}$, J be mappings of $\mathrm{X}$ into $\mathcal{B}(\mathrm{X})$ which satisfy conditions (3.1) and (3.2). Then

(a) $\mathrm{V}_{\mathrm{n}}\left(\mathrm{Z}_{\mathrm{n}+2}\right)=0 \forall \mathrm{n} \in \mathbb{N}$;

(b) $\left\{\mathrm{V}_{\mathrm{n}}(\mathrm{C})\right\}$ is a non-increasing sequence of non-negative real numbers.

(c) $\operatorname{Lim}_{\mathrm{n} \rightarrow \infty} \mathrm{V}_{\mathrm{n}}(\mathrm{C})=0$;

(d) $\lim _{\mathrm{i}, \mathrm{j}, \mathrm{k} \rightarrow \infty} \delta\left(\mathrm{Z}_{\mathrm{i}}, \mathrm{Z}_{\mathrm{j}}, \mathrm{Z}_{\mathrm{k}}\right)=0$ for $\mathrm{i}, \mathrm{j}, \mathrm{k} \in \mathrm{N}$, where $\left\{\mathrm{Z}_{\mathrm{n}}\right\}$ is a sequence described by (3.3).

Proof (a) By axiom (M2), we have; $\mathrm{V}_{\mathrm{n}}\left(\mathrm{Z}_{\mathrm{n}}\right)=0=\mathrm{V}_{\mathrm{n}}\left(\mathrm{Z}_{\mathrm{n}+1}\right) \forall \mathrm{n} \in \mathbb{N}$. First, we will prove that $V_{2 n}\left(Z_{2 n+2}\right)=0 \forall n \in \mathbb{N}$. Suppose, to the contrary, that $V_{2 n}\left(Z_{2 n+2}\right) \neq 0$ for some $n \in \mathbb{N}$. Since $\varphi$ is increasing function, from (3.1), we have;

$$
\begin{aligned}
\mathrm{V}_{2 \mathrm{n}}\left(\mathrm{Z}_{2 \mathrm{n}+2}\right) & =\delta\left(\mathrm{Z}_{2 \mathrm{n}}, \mathrm{Z}_{2 \mathrm{n}+1}, \mathrm{Z}_{2 \mathrm{n}+2}\right) \\
& =\delta\left(\mathrm{Z}_{2 \mathrm{n}+2}, \mathrm{Z}_{2 \mathrm{n}+1}, \mathrm{Z}_{2 \mathrm{n}}\right)=\delta\left(\mathrm{Fx}_{2 \mathrm{n}+2}, \mathrm{Gx}_{2 \mathrm{n}+1}, \mathrm{Z}_{2 \mathrm{n}}\right) \\
& \leq \varphi\left(\delta\left(\mathrm{Ix}_{2 \mathrm{n}+2}, \mathrm{Jx}_{2 \mathrm{n}+1}, \mathrm{Z}_{2 \mathrm{n}}\right), \delta\left(\mathrm{Ix}_{2 \mathrm{n}+2}, \mathrm{Fx}_{2 \mathrm{n}+2}, \mathrm{Z}_{2 \mathrm{n}}\right), \delta\left(\mathrm{Jx}_{2 \mathrm{n}+1}, \mathrm{Gx}_{2 \mathrm{n}+1}, \mathrm{Z}_{2 \mathrm{n}}\right)\right. \\
& \left.\mathcal{D}\left(\mathrm{Ix}_{2 \mathrm{n}+2}, \mathrm{Gx}_{2 \mathrm{n}+1}, \mathrm{Z}_{2 \mathrm{n}}\right), \mathcal{D}\left(\mathrm{Jx}_{2 \mathrm{n}+1}, \mathrm{Fx}_{2 \mathrm{n}+2}, \mathrm{Z}_{2 \mathrm{n}}\right)\right) \\
\leq & \varphi\left(\delta\left(\mathrm{Z}_{2 \mathrm{n}+1}, \mathrm{Z}_{2 \mathrm{n}}, \mathrm{Z}_{2 \mathrm{n}}\right), \delta\left(\mathrm{Z}_{2 \mathrm{n}+1}, \mathrm{Z}_{2 \mathrm{n}+2}, \mathrm{Z}_{2 \mathrm{n}}\right), \delta\left(\mathrm{Z}_{2 \mathrm{n}}, \mathrm{Z}_{2 \mathrm{n}+1}, \mathrm{Z}_{2 \mathrm{n}}\right)\right. \\
, & \left.\mathcal{D}\left(\mathrm{Z}_{2 \mathrm{n}+1}, \mathrm{Z}_{2 \mathrm{n}+1}, \mathrm{Z}_{2 \mathrm{n}}\right), \mathcal{D}\left(\mathrm{Z}_{2 \mathrm{n}}, \mathrm{Z}_{2 \mathrm{n}+2}, \mathrm{Z}_{2 \mathrm{n}}\right)\right) \\
\leq & \varphi\left(\mathrm{V}_{2 \mathrm{n}}\left(\mathrm{Z}_{2 \mathrm{n}}\right), \mathrm{V}_{2 \mathrm{n}}\left(\mathrm{Z}_{2 \mathrm{n}+2}\right), \mathrm{V}_{2 \mathrm{n}}\left(\mathrm{Z}_{2 \mathrm{n}}\right), \mathrm{V}_{2 \mathrm{n}}\left(\mathrm{Z}_{2 \mathrm{n}+1}\right), \delta\left(\mathrm{Z}_{2 \mathrm{n}}, \mathrm{Z}_{2 \mathrm{n}+2}, \mathrm{Z}_{2 \mathrm{n}}\right)\right)
\end{aligned}
$$




$$
\begin{aligned}
& \leq \varphi\left(0, V_{2 n}\left(Z_{2 n+2}\right), 0,0,0\right) \\
& \leq \varphi\left(V_{2 n}\left(Z_{2 n+2}\right), V_{2 n}\left(Z_{2 n+2}\right), V_{2 n}\left(Z_{2 n+2}\right), 2 V_{2 n}\left(Z_{2 n+2}\right), V_{2 n}\left(Z_{2 n+2}\right)\right) \\
& =\gamma\left(V_{2 n}\left(Z_{2 n+2}\right)\right)<V_{2 n}\left(Z_{2 n+2}\right)
\end{aligned}
$$

This is a contradiction. Thus, our supposition that $V_{2 n}\left(Z_{2 n+2}\right) \neq 0$ for some $n \in \mathbb{N}$ was wrong. Hence $V_{2 n}\left(Z_{2 n+2}\right)=0$ for $n \in N$. Similarly, one can show that $V_{2 n-1}\left(Z_{2 n+2}\right)=0$ forn $\in \mathbb{N}$. Consequently, $\mathrm{V}_{\mathrm{n}}\left(\mathrm{Z}_{\mathrm{n}+2}\right)=0 \forall \mathrm{n} \in \mathbb{N}$.

Proof (b) First, we will prove that $\mathrm{V}_{2 \mathrm{n}}(\mathrm{C}) \leq \mathrm{V}_{2 \mathrm{n}-1}(\mathrm{C}) \forall \mathrm{n} \in \mathbb{N}$. Suppose, to the contrary, that $V_{2 n}(C)>V_{2 n-1}(C)$ for some $n \in \mathbb{N}$. Since $\varphi$ is increasing function, from (3.1), we have;

$$
\begin{aligned}
\mathrm{V}_{2 \mathrm{n}}(\mathrm{C}) & =\delta\left(\mathrm{Z}_{2 \mathrm{n}}, \mathrm{Z}_{2 \mathrm{n}+1}, \mathrm{C}\right)=\delta\left(\mathrm{Fx}_{2 \mathrm{n}}, \mathrm{Gx}_{2 \mathrm{n}+1}, \mathrm{C}\right) \\
& \leq \varphi\left(\delta\left(\mathrm{Ix}_{2 \mathrm{n}}, \mathrm{Jx}_{2 \mathrm{n}+1}, \mathrm{C}\right), \delta\left(\mathrm{Ix}_{2 \mathrm{n}}, \mathrm{Gx} \mathrm{x}_{2 \mathrm{n}+1}, \mathrm{C}\right), \delta\left(\mathrm{Jx}_{2 \mathrm{n}+1}, \mathrm{Gx}_{2 \mathrm{n}+1}, \mathrm{C}\right)\right. \\
& \left.\mathcal{D}\left(\mathrm{Ix}_{2 \mathrm{n}}, \mathrm{Gx}_{2 \mathrm{n}+1}, \mathrm{C}\right), \mathcal{D}\left(\mathrm{Jx}_{2 \mathrm{n}+1}, \mathrm{Fx}_{2 \mathrm{n}}, \mathrm{C}\right)\right) \\
& \leq \varphi\left(\mathrm{V}_{2 \mathrm{n}-1}(\mathrm{C}), \mathrm{V}_{2 \mathrm{n}-1}(\mathrm{C}), \mathrm{V}_{2 \mathrm{n}}, \mathrm{V}_{2 \mathrm{n}-1}(\mathrm{C}), \mathrm{V}_{2 \mathrm{n}}(\mathrm{C})\right) \\
& \leq \varphi\left(\mathrm{V}_{2 \mathrm{n}}(\mathrm{C}), \mathrm{V}_{2 \mathrm{n}}(\mathrm{C}), 2 \mathrm{~V}_{2 \mathrm{n}}(\mathrm{C}), \mathrm{V}_{2 \mathrm{n}}(\mathrm{C}), \mathrm{V}_{2 \mathrm{n}}(\mathrm{C})\right)=\gamma\left(\mathrm{V}_{2 \mathrm{n}}(\mathrm{C})\right)<\mathrm{V}_{2 \mathrm{n}}(\mathrm{C})
\end{aligned}
$$

This is a contradiction. Thus, our supposition that $V_{2 n}(C)>V_{2 n-1}(C)$ for some $n \in \mathbb{N}$ was wrong. Hence $V_{2 n}(C) \leq V_{2 n-1}(C)$ for $n \in N$. Similarly, one can show that $V_{2 n+1}(C) \leq V_{2 n}(C)$ forn $\in \mathbb{N}$. Consequently, $\left\{\mathrm{V}_{\mathrm{n}}(\mathrm{C})\right\}$ is non-increasing sequence of non-negative real numbers.

Proof (c) Since $\varphi$ is an increasing function and $\max \left\{\mathrm{V}_{0}(\mathrm{C}), \mathrm{V}_{1}(\mathrm{C})\right\}=\mathrm{V}_{0}(\mathrm{C})$, from (3.1), we get;

$$
\begin{aligned}
\mathrm{V}_{1}(\mathrm{C}) & =\delta\left(\mathrm{Z}_{1}, \mathrm{Z}_{2}, \mathrm{C}\right)=\delta\left(\mathrm{Fx}_{1}, \mathrm{Gx}_{2}, \mathrm{C}\right) \\
& \leq \varphi\left(\delta\left(\mathrm{Ix}_{1}, \mathrm{Jx}_{2}, \mathrm{C}\right), \delta\left(\mathrm{Ix}_{1}, \mathrm{Fx}_{1}, \mathrm{C}\right), \delta\left(\mathrm{Jx}_{2}, \mathrm{Gx}_{2}, \mathrm{C}\right), \mathcal{D}\left(\mathrm{Ix}_{1}, \mathrm{Gx}_{2}, \mathrm{C}\right), \mathcal{D}\left(\mathrm{Jx}_{2}, \mathrm{Fx}_{1}, \mathrm{C}\right)\right) \\
& \leq \varphi\left(\delta\left(\mathrm{Ix}_{1}, \mathrm{Jx}_{2}, \mathrm{C}\right), \delta\left(\mathrm{Ix}_{1}, \mathrm{Fx}_{1}, \mathrm{C}\right), \delta\left(\mathrm{Jx}_{2}, \mathrm{Gx}_{2}, \mathrm{C}\right), \mathcal{D}\left(\mathrm{Ix}_{1}, \mathrm{Gx}_{2}, \mathrm{C}\right), \mathcal{D}\left(\mathrm{Jx}_{2}, \mathrm{Fx}_{1}, \mathrm{C}\right)\right) \\
& \leq \varphi\left(\mathrm{V}_{0}(\mathrm{C}), \mathrm{V}_{0}(\mathrm{C}), \mathrm{V}_{1}(\mathrm{C}), \mathrm{V}_{0}(\mathrm{C}), \mathrm{V}_{1}(\mathrm{C})\right) \\
& \leq \varphi\left(\mathrm{V}_{0}(\mathrm{C}), \mathrm{V}_{0}(\mathrm{C}), \mathrm{V}_{0}(\mathrm{C}), \mathrm{V}_{0}(\mathrm{C}), \mathrm{V}_{0}(\mathrm{C})\right) \\
& \leq \varphi\left(\mathrm{V}_{0}(\mathrm{C}), \mathrm{V}_{0}(\mathrm{C}), 2 \mathrm{~V}_{0}(\mathrm{C}), \mathrm{V}_{0}(\mathrm{C}), \mathrm{V}_{0}(\mathrm{C})\right)=\gamma\left(\mathrm{V}_{0}(\mathrm{C})\right)
\end{aligned}
$$

In general, we have $V_{n}(C) \leq \gamma^{n}\left(V_{0}\right)$. So if $V_{0}(C)>0$, then Lemma 1.1 gives $\lim _{n \rightarrow \infty} V_{n}(C)=0$. For $V_{0}(C)=0$, we clearly have $\lim _{n \rightarrow \infty} V_{n}(C)=0$, since then $V_{n}(C)=0$ for each $n$. This means that, for each $n, \lim _{n \rightarrow \infty} V_{n}(C)=0$.

Proof (d) By axiom (M4), we have;

$$
\delta\left(\mathrm{Z}_{\mathrm{i}}, \mathrm{Z}_{\mathrm{j}}, \mathrm{Z}_{\mathrm{k}}\right) \leq \delta\left(\mathrm{Z}_{\mathrm{i}}, \mathrm{Z}_{\mathrm{j}}, \mathrm{C}\right)+\delta\left(\mathrm{Z}_{\mathrm{i}}, \mathrm{C}, \mathrm{Z}_{\mathrm{k}}\right)+\delta\left(\mathrm{C}, \mathrm{Z}_{\mathrm{j}}, \mathrm{Z}_{\mathrm{k}}\right)
$$

Suppose that $\mathrm{i}<j$, then again by axiom $\left(\mathrm{M}_{4}\right)$, we get; for all $\mathrm{C} \in \mathcal{B}(\mathrm{X})$,

$$
\delta\left(\mathrm{Z}_{\mathrm{i}}, \mathrm{Z}_{\mathrm{j}}, \mathrm{C}\right) \leq \mathrm{V}_{\mathrm{i}}\left(\mathrm{Z}_{\mathrm{i}+2}\right)+\mathrm{V}_{\mathrm{i}+1}\left(\mathrm{Z}_{\mathrm{i}+3}\right)+\mathrm{V}_{\mathrm{i}+2}\left(\mathrm{Z}_{\mathrm{i}+4}\right)+\cdots+\mathrm{V}_{\mathrm{j}-2}\left(\mathrm{Z}_{\mathrm{j}}\right)+\mathrm{V}_{\mathrm{j}-1}(\mathrm{C})
$$

Using (a), on taking $\mathrm{i}, \mathrm{j} \rightarrow \infty$ in the above inequality and using (c), we get

$$
\lim _{i, j \rightarrow \infty} \delta\left(Z_{i}, Z_{j}, C\right)=0 .
$$

Similarly, we can show that

$$
\lim _{\mathrm{j}, \mathrm{k} \rightarrow \infty} \delta\left(\mathrm{Z}_{\mathrm{j}}, \mathrm{Z}_{\mathrm{k}}, \mathrm{C}\right)=0 \text { and } \lim _{\mathrm{i}, \mathrm{j} \rightarrow \infty} \delta\left(\mathrm{Z}_{\mathrm{i}}, \mathrm{Z}_{\mathrm{k}}, \mathrm{C}\right)=0
$$


Point Theorems for Non-compatible, Discontinuous Hybrid Pairs of Mappings on 2-Metric Spaces by Using Implicit Relation

On taking $\mathrm{i}, \mathrm{j}, \mathrm{k} \rightarrow \infty$ in $(3.7)$, we obtain that $\lim _{\mathrm{i}, \mathrm{j}, \mathrm{k} \rightarrow \infty} \delta\left(\mathrm{Z}_{\mathrm{i}}, \mathrm{Z}_{\mathrm{j}}, \mathrm{Z}_{\mathrm{k}}\right)=0$.

Appling Lemma 3.1, we prove the following key lemma.

Lemma 3.2 Let $(X, d)$ be a metric space. Let $I, J$ be mappings of $X$ into itself and $\mathrm{F}, \mathrm{G}$ of $\mathrm{X}$ into $\mathcal{B}(\mathrm{X})$ satisfying (3.1) and (3.2). Then the sequence $\left\{\mathrm{Z}_{\mathrm{n}}\right\}$ (described by (3.3)) is a Cauchy sequence in $X$.

Proof Let $\mathrm{z}_{\mathrm{n}}$ is an arbitrary point in the set $\mathrm{Z}_{\mathrm{n}}$ for $\mathrm{n} \in \mathrm{N}$. By lemma 3.1, we have for all $\mathrm{C} \in \mathcal{B}(\mathrm{X})$; $\lim _{n \rightarrow \infty} V_{n}(C)=\lim _{n \rightarrow \infty} \delta\left(Z_{n}, Z_{n+1}, C\right)=0$. Since $\lim _{n \rightarrow \infty} \delta\left(z_{n}, z_{n+1}, C\right) \leq \lim _{n \rightarrow \infty} \delta\left(Z_{n}, Z_{n+1}, C\right)$ $=0$, it is sufficient to show that $\left\{Z_{2 n}\right\}$ is a Cauchy sequence. Suppose to the contrary, that $\left\{Z_{2 n}\right\}$ is not a Cauchy sequence. Thus, assume there exists $\varepsilon>0$ such that for each even integer $2 \mathrm{k}, \mathrm{k} \in \mathbb{N} \cup\{0\}$, even integers $2 \mathrm{~m}_{\mathrm{k}}$ and $2 \mathrm{n}_{\mathrm{k}}$ with $2 \mathrm{k} \leq 2 \mathrm{n}_{\mathrm{k}} \leq 2 \mathrm{~m}_{\mathrm{k}}$ can be found for which

$$
\delta\left(\mathrm{Z}_{2 \mathrm{~m}_{\mathrm{k}}}, \mathrm{Z}_{2 \mathrm{n}_{\mathrm{k}}}, \mathrm{C}\right)>\varepsilon
$$

For each integer $\mathrm{k}$, fix $2 \mathrm{n}_{\mathrm{k}}$ and let $2 \mathrm{~m}_{\mathrm{k}}$ be the least even integer exceeding $2 \mathrm{n}_{\mathrm{k}}$ and satisfying (3.5), then

$$
2 \mathrm{k} \leq 2 \mathrm{n}_{\mathrm{k}}<2 \mathrm{~m}_{\mathrm{k}}, \delta\left(\mathrm{Z}_{2 \mathrm{~m}_{\mathrm{k}}-2}, \mathrm{Z}_{2 \mathrm{n}_{\mathrm{k}}}, \mathrm{C}\right) \leq \varepsilon, \delta\left(\mathrm{Z}_{2 \mathrm{~m}_{\mathrm{k}}}, \mathrm{Z}_{2 \mathrm{n}_{\mathrm{k}}}, \mathrm{C}\right)>\varepsilon
$$

Hence, for each integer 2k, by axiom (M4), we have;

$$
\begin{aligned}
\varepsilon & <\delta\left(\mathrm{Z}_{2 \mathrm{~m}_{\mathrm{k}}}, \mathrm{Z}_{2 \mathrm{n}_{\mathrm{k}}}, \mathrm{C}\right) \\
& \leq \delta\left(\mathrm{Z}_{2 \mathrm{~m}_{\mathrm{k}}-2}, \mathrm{Z}_{2 \mathrm{n}_{\mathrm{k}}}, \mathrm{C}\right)+\delta\left(\mathrm{Z}_{2 \mathrm{~m}_{\mathrm{k}}}, \mathrm{Z}_{2 \mathrm{~m}_{\mathrm{k}}-2}, \mathrm{C}\right)+\delta\left(\mathrm{Z}_{2 \mathrm{~m}_{\mathrm{k}}}, \mathrm{Z}_{2 \mathrm{n}_{\mathrm{k}}}, \mathrm{Z}_{2 \mathrm{~m}_{\mathrm{k}}-2}\right) \\
& \leq \delta\left(\mathrm{Z}_{2 \mathrm{~m}_{\mathrm{k}}-2}, \mathrm{Z}_{2 \mathrm{n}_{\mathrm{k}}}, \mathrm{C}\right)+\delta\left(\mathrm{Z}_{2 \mathrm{~m}_{\mathrm{k}}}, \mathrm{Z}_{2 \mathrm{~m}_{\mathrm{k}}-1}, \mathrm{C}\right)+\delta\left(\mathrm{Z}_{2 \mathrm{~m}_{\mathrm{k}}-1}, \mathrm{Z}_{2 \mathrm{~m}_{\mathrm{k}}-2}, \mathrm{C}\right) \\
& +\delta\left(\mathrm{Z}_{2 \mathrm{~m}_{\mathrm{k}}}, \mathrm{Z}_{2 \mathrm{~m}_{\mathrm{k}}-2}, \mathrm{Z}_{2 \mathrm{~m}_{\mathrm{k}}-1}\right)+\delta\left(\mathrm{Z}_{2 \mathrm{~m}_{\mathrm{k}}}, \mathrm{Z}_{2 \mathrm{n}_{\mathrm{k}}}, \mathrm{Z}_{2 \mathrm{~m}_{\mathrm{k}}-2}\right) \\
& \leq \delta\left(\mathrm{Z}_{2 \mathrm{n}_{\mathrm{k}}}, \mathrm{Z}_{2 \mathrm{~m}_{\mathrm{k}}-2}, \mathrm{C}\right)+\mathrm{V}_{2 \mathrm{~m}_{\mathrm{k}}-1}(\mathrm{C})+\mathrm{V}_{2 \mathrm{~m}_{\mathrm{k}}-2}(\mathrm{C}) \\
& +\mathrm{V}_{2 \mathrm{~m}_{\mathrm{k}}-2}\left(\mathrm{Z}_{2 \mathrm{~m}_{\mathrm{k}}}\right)+\delta\left(\mathrm{Z}_{2 \mathrm{~m}_{\mathrm{k}}}, \mathrm{Z}_{2 \mathrm{n}_{\mathrm{k}}}, \mathrm{Z}_{2 \mathrm{~m}_{\mathrm{k}}-2}\right)
\end{aligned}
$$

On letting $\mathrm{k} \rightarrow+\infty$ in the above inequality, and using Lemma 3.1, we obtain:

$$
\lim _{\mathrm{n} \rightarrow \infty} \delta\left(\mathrm{Z}_{2 \mathrm{~m}_{\mathrm{k}}}, \mathrm{Z}_{2 \mathrm{n}_{\mathrm{k}}}, \mathrm{C}\right)=\varepsilon
$$

Moreover, by axiom $\left(\mathrm{M}_{4}\right)$, we also have

$$
\begin{aligned}
-\mathrm{V}_{2 \mathrm{~m}_{\mathrm{k}}}(\mathrm{C})-\mathrm{V}_{2 \mathrm{n}_{\mathrm{k}}}(\mathrm{C}) & +\delta\left(\mathrm{Z}_{2 \mathrm{~m}_{\mathrm{k}}}, \mathrm{Z}_{2 \mathrm{n}_{\mathrm{k}}}, \mathrm{C}\right) \\
\leq & \delta\left(\mathrm{Z}_{2 \mathrm{n}_{\mathrm{k}}+1}, \mathrm{Z}_{2 \mathrm{~m}_{\mathrm{k}}+1}, \mathrm{C}\right)+\delta\left(\mathrm{Z}_{2 \mathrm{n}_{\mathrm{k}}}, \mathrm{Z}_{2 \mathrm{~m}_{\mathrm{k}}}, \mathrm{Z}_{2 \mathrm{~m}_{\mathrm{k}}+1}\right)+\delta\left(\mathrm{Z}_{2 \mathrm{n}_{\mathrm{k}}}, \mathrm{Z}_{2 \mathrm{~m}_{\mathrm{k}}+1}, \mathrm{Z}_{2 \mathrm{n}_{\mathrm{k}}+1}\right) \\
& \leq \mathrm{V}_{2 \mathrm{n}_{\mathrm{k}}}(\mathrm{C})+\delta\left(\mathrm{Z}_{2 \mathrm{n}_{\mathrm{k}}}, \mathrm{Z}_{2 \mathrm{~m}_{\mathrm{k}}+1}, \mathrm{C}\right)+\delta\left(\mathrm{Z}_{2 \mathrm{n}_{\mathrm{k}}+1}, \mathrm{Z}_{2 \mathrm{~m}_{\mathrm{k}}+1}, \mathrm{Z}_{2 \mathrm{n}_{\mathrm{k}}}\right) \\
& +\delta\left(\mathrm{Z}_{2 \mathrm{n}_{\mathrm{k}}}, \mathrm{Z}_{2 \mathrm{~m}_{\mathrm{k}}}, \mathrm{Z}_{2 \mathrm{~m}_{\mathrm{k}}+1}\right)+\delta\left(\mathrm{Z}_{2 \mathrm{n}_{\mathrm{k}}}, \mathrm{Z}_{2 \mathrm{~m}_{\mathrm{k}}+1}, \mathrm{Z}_{2 \mathrm{n}_{\mathrm{k}}+1}\right) \\
& \leq \mathrm{V}_{2 \mathrm{n}_{\mathrm{k}}}(\mathrm{C})+\delta\left(\mathrm{Z}_{2 \mathrm{n}_{\mathrm{k}}}, \mathrm{Z}_{2 \mathrm{~m}_{\mathrm{k}}}, \mathrm{C}\right)+\mathrm{V}_{2 \mathrm{~m}_{\mathrm{k}}}(\mathrm{C}) \\
& +2 \delta\left(\mathrm{Z}_{2 \mathrm{n}_{\mathrm{k}}}, \mathrm{Z}_{2 \mathrm{~m}_{\mathrm{k}}+1}, \mathrm{Z}_{2 \mathrm{~m}_{\mathrm{k}}}\right)+2 \delta\left(\mathrm{Z}_{2 \mathrm{n}_{\mathrm{k}}+1}, \mathrm{Z}_{2 \mathrm{~m}_{\mathrm{k}}+1}, \mathrm{Z}_{2 \mathrm{n}_{\mathrm{k}}}\right)
\end{aligned}
$$

On letting $\mathrm{k} \rightarrow+\infty$, using (3.10) and Lemma 3.1, we obtain;

$$
\varepsilon \leq \lim _{\mathrm{k} \rightarrow+\infty} \delta\left(\mathrm{Z}_{2 \mathrm{n}_{\mathrm{k}}+1}, \mathrm{Z}_{2 \mathrm{~m}_{\mathrm{k}}+1}, \mathrm{C}\right) \leq \varepsilon
$$

Hence 


\section{R.D. Daheriya et al.}

$$
\lim _{\mathrm{k} \rightarrow+\infty} \delta\left(\mathrm{Z}_{2 \mathrm{n}_{\mathrm{k}}+1}, \mathrm{Z}_{2 \mathrm{~m}_{\mathrm{k}}+1}, \mathrm{C}\right)=\varepsilon
$$

The same argument shows that

$$
\begin{aligned}
& \delta\left(\mathrm{Z}_{2 \mathrm{~m}_{\mathrm{k}}+1}, \mathrm{Z}_{2 \mathrm{n}_{\mathrm{k}}+1}, \mathrm{C}\right)-\mathrm{V}_{2 \mathrm{n}_{\mathrm{k}}}(\mathrm{C}) \\
& \quad \leq \delta\left(\mathrm{Z}_{2 \mathrm{~m}_{\mathrm{k}}+1}, \mathrm{Z}_{2 \mathrm{n}_{\mathrm{k}}}, \mathrm{C}\right)+\delta\left(\mathrm{Z}_{2 \mathrm{~m}_{\mathrm{k}}+1}, \mathrm{Z}_{2 \mathrm{n}_{\mathrm{k}}+1}, \mathrm{Z}_{2 \mathrm{n}_{\mathrm{k}}}\right) \\
& \quad \leq \delta\left(\mathrm{Z}_{2 \mathrm{~m}_{\mathrm{k}}}, \mathrm{Z}_{2 \mathrm{n}_{\mathrm{k}}}, \mathrm{C}\right)+\mathrm{V}_{2 \mathrm{~m}_{\mathrm{k}}}(\mathrm{C})+\delta\left(\mathrm{Z}_{2 \mathrm{~m}_{\mathrm{k}}+1}, \mathrm{Z}_{2 \mathrm{n}_{\mathrm{k}}}, \mathrm{Z}_{2 \mathrm{~m}_{\mathrm{k}}}\right)+\delta\left(\mathrm{Z}_{2 \mathrm{~m}_{\mathrm{k}}+1}, \mathrm{Z}_{2 \mathrm{n}_{\mathrm{k}}+1}, \mathrm{Z}_{2 \mathrm{n}_{\mathrm{k}}}\right)
\end{aligned}
$$

and

$$
\begin{aligned}
\delta\left(\mathrm{Z}_{2 \mathrm{~m}_{\mathrm{k}}+1}, \mathrm{Z}_{2 \mathrm{n}_{\mathrm{k}}+1}, \mathrm{C}\right)-\mathrm{V}_{2 \mathrm{~m}_{\mathrm{k}}}(\mathrm{C}) \leq & \delta\left(\mathrm{Z}_{2 \mathrm{~m}_{\mathrm{k}}}, \mathrm{Z}_{2 \mathrm{n}_{\mathrm{k}}+1}, \mathrm{C}\right)+\delta\left(\mathrm{Z}_{2 \mathrm{~m}_{\mathrm{k}}+1}, \mathrm{Z}_{2 \mathrm{n}_{\mathrm{k}}+1}, \mathrm{Z}_{2 \mathrm{~m}_{\mathrm{k}}}\right) \\
& \leq \delta\left(\mathrm{Z}_{2 \mathrm{~m}_{\mathrm{k}}}, \mathrm{Z}_{2 \mathrm{n}_{\mathrm{k}}}, \mathrm{C}\right)+\mathrm{V}_{2 \mathrm{n}_{\mathrm{k}}}(\mathrm{C})+\delta\left(\mathrm{Z}_{2 \mathrm{~m}_{\mathrm{k}}}, \mathrm{Z}_{2 \mathrm{n}_{\mathrm{k}}+1}, \mathrm{Z}_{2 \mathrm{n}_{\mathrm{k}}}\right) \\
& +\delta\left(\mathrm{Z}_{2 \mathrm{~m}_{\mathrm{k}}+1}, \mathrm{Z}_{2 \mathrm{n}_{\mathrm{k}}+1}, \mathrm{Z}_{2 \mathrm{n}_{\mathrm{k}}}\right)
\end{aligned}
$$

On letting $\mathrm{k} \rightarrow+\infty$ in the above inequalities, and using Lemma 3.1, and (3.10), (3.11), we obtain:

$$
\lim _{\mathrm{k} \rightarrow+\infty} \delta\left(\mathrm{Z}_{2 \mathrm{~m}_{\mathrm{k}}+1}, \mathrm{Z}_{2 \mathrm{n}_{\mathrm{k}}}, \mathrm{C}\right)=\varepsilon, \quad \lim _{\mathrm{k} \rightarrow+\infty} \delta\left(\mathrm{Z}_{2 \mathrm{~m}_{\mathrm{k}}}, \mathrm{Z}_{2 \mathrm{n}_{\mathrm{k}}+1}, \mathrm{C}\right)=\varepsilon .
$$

On the other hand, by assumption (3.1),

$$
\begin{aligned}
\delta\left(\mathrm{Z}_{2 \mathrm{n}_{\mathrm{k}}+1}, \mathrm{Z}_{2 \mathrm{~m}_{\mathrm{k}}+1}, \mathrm{C}\right) & =\delta\left(\mathrm{Fx}_{2 \mathrm{n}_{\mathrm{k}}+1}, \mathrm{Gx}_{2 \mathrm{~m}_{\mathrm{k}}+1}, \mathrm{C}\right) \\
\leq & \varphi\left(\delta\left(\mathrm{Ix}_{2 \mathrm{n}_{\mathrm{k}}+1}, \mathrm{Jx}_{2 \mathrm{~m}_{\mathrm{k}}+1}, \mathrm{C}\right), \delta\left(\mathrm{Ix}_{2 \mathrm{n}_{\mathrm{k}}+1}, \mathrm{Fx}_{2 \mathrm{n}_{\mathrm{k}}+1}, \mathrm{C}\right), \delta\left(\mathrm{Jx}_{2 \mathrm{~m}_{\mathrm{k}}+1}, \mathrm{Gx}_{2 \mathrm{~m}_{\mathrm{k}}+1}, \mathrm{C}\right)\right. \\
, & \left.\mathcal{D}\left(\mathrm{Ix}_{2 \mathrm{n}_{\mathrm{k}}+1}, \mathrm{Gx}_{2 \mathrm{~m}_{\mathrm{k}}+1}, \mathrm{C}\right), \mathcal{D}\left(\mathrm{Jx}_{2 \mathrm{~m}_{\mathrm{k}}+1}, \mathrm{Fx}_{2 \mathrm{n}_{\mathrm{k}}+1}, \mathrm{C}\right)\right) \\
\leq & \varphi \delta\left(\mathrm{Z}_{2 \mathrm{n}_{\mathrm{k}}}, \mathrm{Z}_{2 \mathrm{~m}_{\mathrm{k}}}, \mathrm{C}\right), \delta\left(\mathrm{Z}_{2 \mathrm{n}_{\mathrm{k}}}, \mathrm{Z}_{2 \mathrm{n}_{\mathrm{k}}+1}, \mathrm{C}\right), \delta\left(\mathrm{Z}_{2 \mathrm{~m}_{\mathrm{k}}}, \mathrm{Z}_{2 \mathrm{~m}_{\mathrm{k}}+1}, \mathrm{C}\right) \\
, & \left.\mathcal{D}\left(\mathrm{Z}_{2 \mathrm{n}_{\mathrm{k}}}, \mathrm{Z}_{2 \mathrm{~m}_{\mathrm{k}}+1}, \mathrm{C}\right), \mathcal{D}\left(\mathrm{Z}_{2 \mathrm{~m}_{\mathrm{k}}}, \mathrm{Z}_{2 \mathrm{n}_{\mathrm{k}}+1}, \mathrm{C}\right)\right) \\
\leq & \left.\varphi \delta\left(\mathrm{Z}_{2 \mathrm{n}_{\mathrm{k}}}, \mathrm{Z}_{2 \mathrm{~m}_{\mathrm{k}}}, \mathrm{C}\right), \mathrm{V}_{2 \mathrm{n}_{\mathrm{k}}}(\mathrm{C}), \mathrm{V}_{2 \mathrm{~m}_{\mathrm{k}}}(\mathrm{C}), \delta\left(\mathrm{Z}_{2 \mathrm{n}_{\mathrm{k}}}, \mathrm{Z}_{2 \mathrm{~m}_{\mathrm{k}}+1}, \mathrm{C}\right), \delta\left(\mathrm{Z}_{2 \mathrm{~m}_{\mathrm{k}}}, \mathrm{Z}_{2 \mathrm{n}_{\mathrm{k}}+1}, \mathrm{C}\right)\right)
\end{aligned}
$$

On letting $\mathrm{k} \rightarrow+\infty$ in the above inequality, and using (3.10), (3.11), (3.12) and Lemma 3.1, we obtain; $\varepsilon \leq \varphi(\varepsilon, 0,0, \varepsilon, \varepsilon) \leq \varphi(\varepsilon, \varepsilon, 2 \varepsilon, \varepsilon, \varepsilon)=\gamma(\varepsilon)<\varepsilon$, we reach a contradiction. Thus, our assumption that $\left\{\mathrm{Z}_{2 \mathrm{n}}\right\}$ is not a Cauchy sequence was wrong. Hence $\left\{\mathrm{Z}_{2 \mathrm{n}}\right\}$ is a Cauchy sequence.

Applying proposition 3.1, Lemma 3.1, and Lemma 3.2, we prove the following common result.

Theorem 3.1 Let (X, d) be a metric space. Let I, J be mappings of X into itself and F, G of X into $\mathcal{B}(\mathrm{X})$ satisfying the conditions (3.1) and (3.2). Suppose that one of $\mathrm{I}(\mathrm{X})$ or $\mathrm{J}(\mathrm{X})$ is complete. Then $\mathcal{C}[\mathrm{I}, \mathrm{F}] \neq$ $\varnothing$ and $\mathcal{C}[\mathrm{J}, \mathrm{G}] \neq \varnothing$. Further, if the hybrid pair $\{\mathrm{F}, \mathrm{I}\}$ and $\{\mathrm{G}, \mathrm{J}\}$ are weakly commuting of type (KB) at coincidence points in $\mathrm{X}$, then the set $(\mathcal{F}[\mathrm{I}] \cap \mathcal{F}[J] \cap \mathcal{F}[\mathrm{F}] \cap \mathcal{F}[\mathrm{G}])$ is a singleton set.

Proof Let $\mathrm{Z}_{\mathrm{n}}$ is an arbitrary point in the set $\mathrm{Z}_{\mathrm{n}}$ for $\mathrm{n} \in \mathrm{N}$. By lemma 3.2, the sequence $\left\{\mathrm{Z}_{\mathrm{n}}\right\}$ defined by (3.3) is a Cauchy sequence and hence any subsequence thereof is a Cauchy Sequence in $X$. Suppose that $J(X)$ is a complete subspace of $X$. Since $J x_{2 n+1} \in \mathrm{Fx}_{2 n}=Z_{2 n}$, for $n \in \mathbb{N} \cup\{0\}$,

$$
\delta\left(\mathrm{Jx}_{2 \mathrm{~m}+1}, \mathrm{Jx}_{2 \mathrm{n}+1}, \mathrm{C}\right)=\delta\left(\mathrm{Z}_{2 \mathrm{~m}}, \mathrm{Z}_{2 \mathrm{n}}, \mathrm{C}\right)<\epsilon
$$

for all $C \in \mathcal{B}(X)$ and for $m, n \geq n_{0}, n_{0} \in N$. Therefore $\left\{\mathrm{Jx}_{2 \mathrm{n}+1}\right\}$ is Cauchy and hence $\mathrm{Jx}_{2 \mathrm{n}+1} \rightarrow \mathrm{z}=$ $\mathrm{Jv} \in \mathrm{J}(\mathrm{X})$, for some $\mathrm{v} \in \mathrm{X}$. But $\mathrm{Ix}_{2 \mathrm{n}} \in \mathrm{GX}_{2 \mathrm{n}-1}=\mathrm{Z}_{2 \mathrm{n}-1}$ and hence, we have

$$
\delta\left(\mathrm{Ix}_{2 \mathrm{n}}, \mathrm{Jx}_{2 \mathrm{n}+1}, \mathrm{C}\right)=\delta\left(\mathrm{Z}_{2 \mathrm{n}-1}, \mathrm{Z}_{2 \mathrm{n}}, \mathrm{C}\right)=\mathrm{V}_{2 \mathrm{n}-1}(\mathrm{C}) \rightarrow 0,
$$


Point Theorems for Non-compatible, Discontinuous Hybrid Pairs of Mappings on 2-Metric Spaces by Using Implicit Relation

Consequently, $\mathrm{Ix}_{2 \mathrm{n}} \rightarrow \mathrm{z}$. Moreover, we have for $\mathrm{n} \in \mathrm{N}$,

$$
\begin{aligned}
\delta\left(\mathrm{Fx}_{2 \mathrm{n}}, \mathrm{z}, \mathrm{C}\right) & \leq \delta\left(\mathrm{Fx}_{2 \mathrm{n}}, \mathrm{Ix}_{2 \mathrm{n}}, \mathrm{C}\right)+\delta\left(\mathrm{Ix}_{2 \mathrm{n}}, \mathrm{z}, \mathrm{C}\right)+\delta\left(\mathrm{Fx}_{2 \mathrm{n}}, \mathrm{z}, \mathrm{Ix}_{2 \mathrm{n}}\right) \\
& \leq \delta\left(\mathrm{Z}_{2 \mathrm{n}}, \mathrm{Z}_{2 \mathrm{n}-1}, \mathrm{C}\right)+\mathrm{d}\left(\mathrm{Z}_{2 \mathrm{n}-1}, \mathrm{z}, \mathrm{C}\right)+\delta\left(\mathrm{Z}_{2 \mathrm{n}}, \mathrm{z}, \mathrm{Z}_{2 \mathrm{n}-1}\right)
\end{aligned}
$$

On taking $\mathrm{n} \rightarrow+\infty$ in above inequality, we get;

$$
\delta\left(\mathrm{Fx}_{2 \mathrm{n}}, \mathrm{z}, \mathrm{C}\right) \rightarrow 0
$$

Similarly,

$$
\delta\left(\mathrm{Gx}_{2 \mathrm{n}-1}, \mathrm{z}, \mathrm{C}\right) \rightarrow 0 \text { as } \mathrm{n} \rightarrow \infty .
$$

Since $\varphi$ is an increasing function, by (3.1), we have for $\mathrm{n} \in \mathrm{N}$;

(3.17) $\delta\left(\mathrm{Fx}_{2 n}, \mathrm{Gv}, \mathrm{C}\right) \leq \varphi\left(\delta\left(\mathrm{Ix}_{2 n}, \mathrm{Jv}, \mathrm{C}\right), \delta\left(\mathrm{Ix}_{2 n}, \mathrm{Fx}_{2 n}, \mathrm{C}\right), \delta(\mathrm{Jv}, \mathrm{Gv}, \mathrm{C}), \mathcal{D}\left(\mathrm{Ix}_{2 n}, \mathrm{Gv}, \mathrm{C}\right), \mathcal{D}\left(\mathrm{Jv}, \mathrm{Fx}_{2 n}, \mathrm{C}\right)\right)$

$$
\leq \varphi\left(\delta\left(\mathrm{Z}_{2 \mathrm{n}-1}, \mathrm{Jv}, \mathrm{C}\right), \delta\left(\mathrm{Z}_{2 \mathrm{n}-1}, \mathrm{Z}_{2 \mathrm{n}}, \mathrm{C}\right), \delta(\mathrm{Jv}, \mathrm{Gv}, \mathrm{C}), \delta\left(\mathrm{Z}_{2 \mathrm{n}-1}, \mathrm{Gv}, \mathrm{C}\right), \delta\left(\mathrm{Jv}, \mathrm{Z}_{2 \mathrm{n}}, \mathrm{C}\right)\right)
$$

Since $\delta\left(\mathrm{Z}_{2 \mathrm{n}-1}, \mathrm{Gv}, \mathrm{C}\right) \rightarrow \delta(\mathrm{z}, \mathrm{Gv}, \mathrm{C})$, when $\mathrm{Ix}_{2 \mathrm{n}} \rightarrow \mathrm{z}$. On taking $\mathrm{n} \rightarrow \infty$ in (3.13), we get;

$$
\begin{aligned}
\delta(\mathrm{z}, \mathrm{Gv}, \mathrm{C}) & \leq \varphi(0,0, \delta(\mathrm{z}, \mathrm{Gv}, \mathrm{C}), \delta(\mathrm{z}, \mathrm{Gv}, \mathrm{C}), 0) \\
& \leq \varphi(\delta(\mathrm{z}, \mathrm{Gv}, \mathrm{C}), \delta(\mathrm{z}, \mathrm{Gv}, \mathrm{C}), 2 \delta(\mathrm{z}, \mathrm{Gv}, \mathrm{C}), \delta(\mathrm{z}, \mathrm{Gv}, \mathrm{C}), \delta(\mathrm{z}, \mathrm{Gv}, \mathrm{C})) \\
& =\gamma(\delta(\mathrm{z}, \mathrm{Gv}, \mathrm{C}))<\delta(\mathrm{z}, \mathrm{Gv}, \mathrm{C})
\end{aligned}
$$

a contradiction. Thus $\mathrm{Gv}=\{\mathrm{z}\}=\{\mathrm{Jv}\}$ and so $\mathcal{C}[\mathrm{J}, \mathrm{G}] \neq \emptyset$.But $\mathrm{U} \mathrm{G}(\mathrm{X}) \subseteq \mathrm{I}(\mathrm{X})$, there exists $\mathrm{u} \in \mathrm{X}$ such that $\{\mathrm{Iu}\}=\mathrm{Gv}=\{\mathrm{Jv}\}=\{\mathrm{z}\}$. Now if $\mathrm{Fu} \neq \mathrm{Gv}$, then by (3.1), we have;

$$
\begin{aligned}
\delta(\mathrm{Fu}, \mathrm{Gv}, \mathrm{C}) & \leq \varphi(\delta(\mathrm{Iu}, \mathrm{Jv}, \mathrm{C}), \delta(\mathrm{Iu}, \mathrm{Fu}, \mathrm{C}), \delta(\mathrm{Jv}, \mathrm{Gv}, \mathrm{C}), \mathcal{D}(\mathrm{Iu}, \mathrm{Gv}, \mathrm{C}), \mathcal{D}(\mathrm{Jv}, \mathrm{Fu}, \mathrm{C})) \\
& \leq \varphi(\delta(\mathrm{Iu}, \mathrm{Jv}, \mathrm{C}), \delta(\mathrm{Iu}, \mathrm{Fu}, \mathrm{C}), \delta(\mathrm{Jv}, \mathrm{Gv}, \mathrm{C}), \delta(\mathrm{Iu}, \mathrm{Gv}, \mathrm{C}), \delta(\mathrm{Jv}, \mathrm{Fu}, \mathrm{C})) \\
& =\varphi(0, \delta(\mathrm{Gv}, \mathrm{Fu}, \mathrm{C}), 0,0, \delta(\mathrm{Gv}, \mathrm{Fu}, \mathrm{C})) \\
& \leq \varphi \delta(\mathrm{Fu}, \mathrm{Gv}, \mathrm{C}), \delta(\mathrm{Fu}, \mathrm{Gv}, \mathrm{C}), 2 \delta(\mathrm{Gv}, \mathrm{Fu}, \mathrm{C}), \delta(\mathrm{Gv}, \mathrm{Fu}, \mathrm{C}), \delta(\mathrm{Gv}, \mathrm{Fu}, \mathrm{C})) \\
& =\gamma(\delta(\mathrm{Fu}, \mathrm{Gv}, \mathrm{C}))<\delta(\mathrm{Fu}, \mathrm{Gv}, \mathrm{C})
\end{aligned}
$$

This is a contradiction. Thus, $\mathrm{Fu}=\{\mathrm{Iu}\}=\{\mathrm{Jv}\}=\mathrm{Gv}=\{\mathrm{z}\}$ and so $\mathcal{C}[\mathrm{I}, \mathrm{F}] \neq \varnothing$. Since $\mathrm{Fu}=\{\mathrm{Iu}\}$ and the pair $\{\mathrm{F}, \mathrm{I}\}$ is weakly commuting of type $(\mathrm{KB})$ at coincidence points in $\mathrm{X}$, we obtain $\delta(\mathrm{IIu}, \mathrm{FIu}, \mathrm{C}) \leq \mathrm{R} \delta(\mathrm{Iu}, \mathrm{Fu}, \mathrm{C})$ which gives $\{\mathrm{Iz}\}=\mathrm{Fz}$.

Again since $\mathrm{Gv}=\{\mathrm{Jv}\}$ and the pair $\{\mathrm{G}, \mathrm{J}\}$ is weakly commuting of type (MD) at coincidence points in $\mathrm{X}$, we obtain $\delta(J J v, G J v, C) \leq \mathrm{R} \delta(J v, G v, C)$. This leads to $\{J z\}=G z$. Now, we will prove that $\{\mathrm{z}\}=$ $\mathrm{Fz}=\{\mathrm{Iz}\}=\{\mathrm{Jz}\}=\mathrm{Gz}$. By (3.1), we have

$$
\begin{aligned}
\delta(\mathrm{Fz}, \mathrm{z}, \mathrm{C}) & \leq \delta(\mathrm{Fz}, \mathrm{Gv}, \mathrm{C}) \\
& \leq \varphi(\delta(\mathrm{Iz}, \mathrm{Jv}, \mathrm{C}), \delta(\mathrm{Iz}, \mathrm{Fz}, \mathrm{C}), \delta(\mathrm{Jv}, \mathrm{Gv}, \mathrm{C}), \mathcal{D}(\mathrm{Iz}, \mathrm{Gv}, \mathrm{C}), \mathcal{D}(\mathrm{Jv}, \mathrm{Fz}, \mathrm{C})) \\
& \leq \varphi(\delta(\mathrm{Fz}, \mathrm{z}, \mathrm{C}), 0,0, \delta(\mathrm{Fz}, \mathrm{z}, \mathrm{C}), \delta(\mathrm{z}, \mathrm{Fz}, \mathrm{C})) \\
& \leq \varphi(\delta(\mathrm{Fz}, \mathrm{z}, \mathrm{C}), \delta(\mathrm{Fz}, \mathrm{z}, \mathrm{C}), 2 \delta(\mathrm{Fz}, \mathrm{z}, \mathrm{C}), \delta(\mathrm{Fz}, \mathrm{z}, \mathrm{C}), \delta(\mathrm{Fz}, \mathrm{z}, \mathrm{C})) \\
& =\gamma(\delta(\mathrm{Fz}, \mathrm{z}, \mathrm{C}))<\delta(\mathrm{Fz}, \mathrm{z}, \mathrm{C})
\end{aligned}
$$




\section{R.D. Daheriya et al.}

Here we reach a contradiction if $\delta(\mathrm{Fz}, \mathrm{z}, \mathrm{C})>0$. Thus $\mathrm{Fz}=\{\mathrm{z}\}$. Consequently, we have $\{\mathrm{z}\}=\{\mathrm{Iz}\}=$ Fz . Again by (3.1), we have

$$
\begin{aligned}
\delta(\mathrm{z}, \mathrm{Gz}, \mathrm{C}) & \leq \delta(\mathrm{Fu}, \mathrm{Gz}, \mathrm{C}) \\
& \leq \varphi(\mathrm{d}(\mathrm{Iu}, \mathrm{Jz}, \mathrm{C}), \delta(\mathrm{Iu}, \mathrm{Fu}, \mathrm{C}), \delta(\mathrm{Jz}, \mathrm{Gz}, \mathrm{C}), \mathcal{D}(\mathrm{Iu}, \mathrm{Gz}, \mathrm{C}), \mathcal{D}(\mathrm{Jz}, \mathrm{Fu}, \mathrm{C})) \\
& \leq \varphi(\delta(\mathrm{z}, \mathrm{Gz}, \mathrm{C}), 0,0, \delta(\mathrm{z}, \mathrm{Gz}, \mathrm{C}), \delta(\mathrm{Gz}, \mathrm{z}, \mathrm{C})) \\
& \leq \varphi(\delta(\mathrm{z}, \mathrm{Gz}, \mathrm{C}), \delta(\mathrm{z}, \mathrm{Gz}, \mathrm{C}), 2 \delta(\mathrm{z}, \mathrm{Gz}, \mathrm{C}), \delta(\mathrm{z}, \mathrm{Gz}, \mathrm{C}), \delta(\mathrm{z}, \mathrm{Gz}, \mathrm{C})) \\
& =\gamma(\delta(\mathrm{z}, \mathrm{Gz}, \mathrm{C}))<\delta(\mathrm{z}, \mathrm{Gz}, \mathrm{C})
\end{aligned}
$$

Also, we reach a contradiction if $\delta(\mathrm{z}, \mathrm{Gz}, \mathrm{C})>0$. Thus $\{\mathrm{z}\}=\mathrm{Fz}=\{\mathrm{Iz}\}=\{\mathrm{Jz}\}=\mathrm{Gz}$ and so $(\mathcal{F}[\mathrm{I}] \cap$ $\mathcal{F J} \cap \mathcal{F F} \cap \mathcal{F G} \neq \varnothing$. In view of proposition 3.1, the set $\mathcal{F I} \cap \mathcal{F J} \cap \mathcal{F F} \cap \mathcal{F G}$ is a singleton. If one assumes that $\mathrm{I}(\mathrm{X})$ is a complete subspace of $\mathrm{X}$, then analogous arguments establish that $\mathcal{C}[\mathrm{I}, \mathrm{F}] \neq \varnothing, \mathcal{C}[\mathrm{J}, \mathrm{G}] \neq$ $\varnothing$ and the set $(\mathcal{F}[\mathrm{I}] \cap \mathcal{F}[\mathrm{J}] \cap \mathcal{F}[\mathrm{F}] \cap \mathcal{F}[\mathrm{G}])$ is a singleton. This finishes the proof.

Now, if we put $\mathrm{F}=\mathrm{G}$ and $\mathrm{I}=\mathrm{J}$ in Theorem 3.1, then we obtain the following Corollary.

Corollary: 3.1 Let $(X, d)$ be a 2-metric space. Let I be a mappings of $X$ into itself and $\mathrm{F}$ of $\mathrm{X}$ into $\mathcal{B}(\mathrm{X})$ satisfying the following conditions:

$$
\delta(\mathrm{Fx}, \mathrm{Fy}, \mathrm{C}) \leq \varphi(\delta(\mathrm{Ix}, \mathrm{Iy}, \mathrm{C}), \delta(\mathrm{Ix}, \mathrm{Fx}, \mathrm{C}), \delta(\mathrm{Iy}, \mathrm{Fy}, \mathrm{C}), \mathcal{D}(\mathrm{Ix}, \mathrm{Fy}, \mathrm{C}), \mathcal{D}(\mathrm{Iy}, \mathrm{Fy}, \mathrm{C}))
$$

for all $\mathrm{x}, \mathrm{y} \in \mathrm{X}$. where $\varphi \in \Phi$ and

$$
\mathrm{U} F(\mathrm{X}) \subseteq \mathrm{I}(\mathrm{X})
$$

Suppose that $\mathrm{I}(\mathrm{X})$ is complete. Then $\mathcal{C}[\mathrm{I}, \mathrm{F}] \neq \emptyset$. Further, if the hybrid pair $\{\mathrm{F}, \mathrm{I}\}$ is weakly commuting of type $(\mathrm{KB})$ at coincidence points in $\mathrm{X}$, then the set $(\mathcal{F}[\mathrm{I}] \cap \mathcal{F}[\mathrm{F}])$ is a singleton.

If we put $\mathrm{I}=\mathrm{J}$ in Theorem 3.1, then we obtain the following Corollary.

Corollary 3.2 Let $(\mathrm{X}, \mathrm{d})$ be a 2-metric space. Let $\mathrm{I}$ be a mapping of $\mathrm{X}$ into itself and $\mathrm{F}, \mathrm{G}$ of $\mathrm{X}$ into $\mathcal{B}(\mathrm{X})$ satisfying the following conditions:

$$
\delta(\mathrm{Fx}, \mathrm{Gy}, \mathrm{C}) \leq \varphi(\delta(\mathrm{Ix}, \mathrm{Iy}, \mathrm{C}), \delta(\mathrm{Ix}, \mathrm{Fx}, \mathrm{C}), \delta(\mathrm{Iy}, \mathrm{Gy}, \mathrm{C}), \mathcal{D}(\mathrm{Ix}, \mathrm{Gy}, \mathrm{C}), \mathcal{D}(\mathrm{Iy}, \mathrm{Fx}, \mathrm{C}))
$$

for all $\mathrm{x}, \mathrm{y} \in \mathrm{X}$. where $\varphi \in \Phi$ and

$$
\mathrm{UF}(\mathrm{X}) \subseteq \mathrm{I}(\mathrm{X}) \text { and } \mathrm{U} \mathrm{F}(\mathrm{X}) \subseteq \mathrm{I}(\mathrm{X}) \text {. }
$$

Suppose that $\mathrm{I}(\mathrm{X})$ is complete. Then $\mathcal{C}[\mathrm{I}, \mathrm{F}] \neq \varnothing$ and $\mathcal{C}[\mathrm{I}, \mathrm{G}] \neq \varnothing$. Further, if the hybrid pairs $\{\mathrm{F}, \mathrm{I}\}$ and $\{\mathrm{G}, \mathrm{I}\}$ are weakly commuting of type $(\mathrm{KB})$ at coincidence points in $\mathrm{X}$, then the set $(\mathcal{F}[\mathrm{I}] \cap$ $\mathcal{F F} \cap \mathcal{F G}$ is a singleton.

If we put $F=G$ in Theorem 3.1, then we obtain the following Corollary.

Corollary 3.3 Let $(\mathrm{X}, \mathrm{d})$ be a 2-metric space. Let I, J be a mappings of $\mathrm{X}$ into itself and $\mathrm{F}$ of $\mathrm{X}$ into $\mathcal{B}(\mathrm{X})$ satisfying the following conditions:

$$
\delta(\mathrm{Fx}, \mathrm{Fy}, \mathrm{C}) \leq \varphi(\delta(\mathrm{Ix}, \mathrm{Jy}, \mathrm{C}), \delta(\mathrm{Ix}, \mathrm{Fx}, \mathrm{C}), \delta(\mathrm{Jy}, \mathrm{Fy}, \mathrm{C}), \mathcal{D}(\mathrm{Ix}, \mathrm{Fy}, \mathrm{C}), \mathcal{D}(\mathrm{Jy}, \mathrm{Fx}, \mathrm{C}))
$$

for all $\mathrm{x}, \mathrm{y} \in \mathrm{X}$, where $\varphi \in \Phi$ and

$$
\mathrm{U} F(X) \subseteq \mathrm{I}(\mathrm{X}) \cap \mathrm{J}(\mathrm{X}) .
$$


Point Theorems for Non-compatible, Discontinuous Hybrid Pairs of Mappings on 2-Metric Spaces by Using Implicit Relation

Suppose that one of $\mathrm{I}(\mathrm{X})$ or $\mathrm{J}(\mathrm{X})$ is complete. Then $\mathcal{C}[\mathrm{I}, \mathrm{F}] \neq \varnothing$ and $\mathcal{C}[\mathrm{J}, \mathrm{F}] \neq \varnothing$. Further, if the hybrid pair $\{\mathrm{F}, \mathrm{I}\}$ and $\{\mathrm{F}, \mathrm{J}\}$ are weakly commuting of type $(\mathrm{KB})$ at coincidence points in $\mathrm{X}$, then the set $(\mathcal{F}[\mathrm{I}] \cap \mathcal{F}[\mathrm{J}] \cap \mathcal{F}[\mathrm{F}])$ is a singleton.

Corollary: 3.4 Let $(\mathrm{X}, \mathrm{d})$ be a 2-metric space. Let $\mathrm{I}, \mathrm{J}, \mathrm{S}, \mathrm{T}$ be mappings of $\mathrm{X}$ into itself and $\mathrm{F}, \mathrm{G}$ of $\mathrm{X}$ into $\mathcal{B}(\mathrm{X})$ satisfying the following conditions:

$$
\delta(\mathrm{Fx}, \mathrm{Gy}, \mathrm{C}) \leq \varphi(\delta(\mathrm{ISx}, \mathrm{JTy}, \mathrm{C}), \delta(\mathrm{Fx}, \mathrm{ISx}, \mathrm{C}), \delta(\mathrm{Gy}, \mathrm{JTy}, \mathrm{C}), \mathcal{D}(\mathrm{Gy}, \mathrm{ISx}, \mathrm{C}), \mathcal{D}(\mathrm{Fx}, \mathrm{JTy}, \mathrm{C}))
$$

$\forall \mathrm{x}, \mathrm{y} \in \mathrm{X}$ and $\forall \mathrm{C} \in \mathrm{B}(\mathrm{X})$, where $\varphi \in \Phi$. Suppose

$$
\mathrm{U} F(\mathrm{X}) \subseteq \mathrm{JT}(\mathrm{X}) \text { and } \mathrm{U} \mathrm{G}(\mathrm{X}) \subseteq \mathrm{IS}(\mathrm{X}) \text {, }
$$

Suppose that one of IS $(\mathrm{X})$ or JT(X) is a complete subspace of $\mathrm{X}$ and both the hybrid pairs $\{\mathrm{F}, \mathrm{IS}\}$ and $\{G, J T\}$ are weakly commuting of type $(\mathrm{KB})$ at coincidence points in $\mathrm{X}$. If IS $=\mathrm{SI}$, JT $=\mathrm{TJ}, \mathrm{FS}=$ $\mathrm{SF}, \mathrm{GT}=\mathrm{TG}$, then the set $(\mathcal{F}[\mathrm{I}] \cap \mathcal{F}[\mathrm{J}] \cap \mathcal{F}[\mathrm{S}] \cap \mathcal{F}[\mathrm{T}] \cap \mathcal{F}[\mathrm{F}] \cap \mathcal{F}[\mathrm{G}])$ is a singleton.

Proof By Theorem 3.1, the set $(\mathcal{F}[\mathrm{IS}] \cap \mathcal{F}[\mathrm{JT}] \cap \mathcal{F}[\mathrm{F}] \cap \mathcal{F}[\mathrm{G}])$ is a singleton set, that is, there exists $\mathrm{z} \in \mathrm{X}$ such that $\{\mathrm{z}\}=\{\mathrm{ISz}\}=\{\mathrm{JTz}\}=\mathrm{Fz}=\mathrm{Gz}$.

We will prove that $S z=z$. If $S z \neq z$, then by (3.21), we have for all $C \in B(X)$,

$$
\begin{aligned}
\delta(\mathrm{FSz}, \mathrm{Gz}, \mathrm{C}) & \leq \varphi(\delta(\mathrm{ISSz}, \mathrm{JTz}, \mathrm{C}), \delta(\mathrm{FSz}, \mathrm{ISSz}, \mathrm{C}), \delta(\mathrm{Gz}, \mathrm{JTz}, \mathrm{C}), \mathcal{D}(\mathrm{Gz}, \mathrm{ISSz}, \mathrm{C}), \mathcal{D}(\mathrm{FSz}, \mathrm{JTz}, \mathrm{C})) \\
\Rightarrow \quad \delta(\mathrm{SFz}, \mathrm{Gz}, \mathrm{C}) & \leq \varphi(\delta(\mathrm{SISz}, \mathrm{JTz}, \mathrm{C}), \delta(\mathrm{SFz}, \mathrm{SISz}, \mathrm{C}), \delta(\mathrm{Gz}, \mathrm{JTz}, \mathrm{C}), \mathcal{D}(\mathrm{Gz}, \mathrm{SISz}, \mathrm{C}), \mathcal{D}(\mathrm{SFz}, \mathrm{JTz}, \mathrm{C})) \\
\Rightarrow \quad \delta(\mathrm{Sz}, \mathrm{z}, \mathrm{C}) & \leq \varphi(\delta(\mathrm{Sz}, \mathrm{z}, \mathrm{C}), \delta(\mathrm{Sz}, \mathrm{Sz}, \mathrm{C}), \delta(\mathrm{z}, \mathrm{z}, \mathrm{C}), \delta(\mathrm{z}, \mathrm{Sz}, \mathrm{C}), \delta(\mathrm{Sz}, \mathrm{z}, \mathrm{C})) \\
& \leq \varphi(\delta(\mathrm{Sz}, \mathrm{z}, \mathrm{C}), 0,0, \delta(\mathrm{z}, \mathrm{Sz}, \mathrm{C}), \delta(\mathrm{Sz}, \mathrm{z}, \mathrm{C})) \\
& \leq \varphi(\delta(\mathrm{Sz}, \mathrm{z}, \mathrm{C}), \delta(\mathrm{Sz}, \mathrm{z}, \mathrm{C}), \delta(\mathrm{Sz}, \mathrm{z}, \mathrm{C}), 2 \delta(\mathrm{Sz}, \mathrm{z}, \mathrm{C}), \delta(\mathrm{Sz}, \mathrm{z}, \mathrm{C})) \\
& =\gamma(\delta(\mathrm{Sz}, \mathrm{z}, \mathrm{C}))<\delta(\mathrm{Sz}, \mathrm{z}, \mathrm{C})
\end{aligned}
$$

This is a contradiction. Thus, our assumption that $\mathrm{Sz} \neq \mathrm{z}$ was wrong and so $\mathrm{Sz}=\mathrm{z}$.

Next, we will prove $\mathrm{Tz}=\mathrm{z}$. On contrary, suppose that $\mathrm{Tz} \neq \mathrm{z}$. By (3.21), we have

$$
\begin{aligned}
\delta(\mathrm{Fz}, \mathrm{GTz}, \mathrm{C}) & \leq \varphi(\delta(\mathrm{ISz}, \mathrm{JTTz}, \mathrm{C}), \delta(\mathrm{Fz}, \mathrm{ISz}, \mathrm{C}), \delta(\mathrm{GTz}, \mathrm{JTTz}, \mathrm{C}), \mathcal{D}(\mathrm{GTz}, \mathrm{ISz}, \mathrm{C}), \mathcal{D}(\mathrm{Fz}, \mathrm{JTTz}, \mathrm{C})) \\
\Rightarrow \quad \delta(\mathrm{Fz}, \mathrm{TGz}, \mathrm{C}) & \leq \varphi(\delta(\mathrm{ISz}, \mathrm{TJTz}, \mathrm{C}), \delta(\mathrm{Fz}, \mathrm{ISz}, \mathrm{C}), \delta(\mathrm{TGz}, \mathrm{TJTz}, \mathrm{C}), \mathcal{D}(\mathrm{TGz}, \mathrm{ISz}, \mathrm{C}), \mathcal{D}(\mathrm{Fz}, \mathrm{TJTz}, \mathrm{C})) \\
\Rightarrow \quad \delta(\mathrm{z}, \mathrm{Tz}, \mathrm{C}) & \leq \varphi(\delta(\mathrm{z}, \mathrm{Tz}, \mathrm{C}), \delta(\mathrm{z}, \mathrm{z}, \mathrm{C}), \delta(\mathrm{Tz}, \mathrm{Tz}, \mathrm{C}), \mathrm{D}(\mathrm{Tz}, \mathrm{z}, \mathrm{C}), \mathrm{D}(\mathrm{z}, \mathrm{Tz}, \mathrm{C})) \\
& \leq \varphi(\delta(\mathrm{z}, \mathrm{Tz}, \mathrm{C}), 0,0, \mathrm{D}(\mathrm{Tz}, \mathrm{z}, \mathrm{C}), \mathrm{D}(\mathrm{z}, \mathrm{Tz}, \mathrm{C})) \\
& \leq \varphi(\delta(\mathrm{z}, \mathrm{Tz}, \mathrm{C}), \delta(\mathrm{z}, \mathrm{Tz}, \mathrm{C}), 2 \delta(\mathrm{z}, \mathrm{Tz}, \mathrm{C}), \delta(\mathrm{z}, \mathrm{Tz}, \mathrm{C}), \delta(\mathrm{z}, \mathrm{Tz}, \mathrm{C})) \\
& =\gamma(\delta(\mathrm{z}, \mathrm{Tz}, \mathrm{C}))<\delta(\mathrm{z}, \mathrm{Tz}, \mathrm{C})
\end{aligned}
$$

a contradiction. Thus, our assumption that $\mathrm{Tz} \neq \mathrm{z}$ was wrong and then $\mathrm{z}=\mathrm{Tz}$. Now, $\mathrm{z}=\mathrm{ISz}=\mathrm{Iz}$ and $\mathrm{z}=\mathrm{JTz}=\mathrm{Jz}$. Hence, $(\mathcal{F}[\mathrm{I}] \cap \mathcal{F}[\mathrm{J}] \cap \mathcal{F}[\mathrm{S}] \cap \mathcal{F}[\mathrm{T}] \cap \mathcal{F}[\mathrm{F}] \cap \mathcal{F}[\mathrm{G}]) \neq \emptyset$. Finally, we will prove that the set $(\mathcal{F}[\mathrm{I}] \cap \mathcal{F}[\mathrm{J}] \cap \mathcal{F}[\mathrm{S}] \cap \mathcal{F}[\mathrm{T}] \cap \mathcal{F}[\mathrm{F}] \cap \mathcal{F}[\mathrm{G}])$ is a singleton. If not, then there exists a pointw $\neq \mathrm{z}$ in X such that $\mathrm{z} \in(\mathcal{F}[\mathrm{I}] \cap \mathcal{F}[\mathrm{J}] \cap \mathcal{F}[\mathrm{S}] \cap \mathcal{F}[\mathrm{T}] \cap \mathcal{F}[\mathrm{F}] \cap \mathcal{F}[\mathrm{G}])$.From (3.21), we have for all $\mathrm{C} \in \mathrm{B}(\mathrm{X})$,

$$
\delta(\mathrm{Fz}, \mathrm{Gw}, \mathrm{C}) \leq \varphi(\delta(\mathrm{ISz}, \mathrm{JTw}, \mathrm{C}), \delta(\mathrm{Fz}, \mathrm{ISz}, \mathrm{C}), \delta(\mathrm{Gw}, \mathrm{JTw}, \mathrm{C}), \mathcal{D}(\mathrm{Gw}, \mathrm{ISz}, \mathrm{C}), \mathcal{D}(\mathrm{Fz}, \mathrm{JTw}, \mathrm{C}))
$$




$$
\begin{aligned}
\Rightarrow \quad \delta(\mathrm{z}, \mathrm{w}, \mathrm{C}) & \leq \varphi(\delta(\mathrm{z}, \mathrm{w}, \mathrm{C}), \delta(\mathrm{z}, \mathrm{z}, \mathrm{C}), \delta(\mathrm{w}, \mathrm{w}, \mathrm{C}), \delta(\mathrm{w}, \mathrm{z}, \mathrm{C}), \delta(\mathrm{z}, \mathrm{w}, \mathrm{C})) \\
& \leq \varphi(\delta(\mathrm{z}, \mathrm{w}, \mathrm{C}), 0,0, \delta(\mathrm{w}, \mathrm{z}, \mathrm{C}), \delta(\mathrm{z}, \mathrm{w}, \mathrm{C})) \\
& \leq \varphi(\delta(\mathrm{z}, \mathrm{w}, \mathrm{C}), \delta(\mathrm{z}, \mathrm{w}, \mathrm{C}), 2 \delta(\mathrm{z}, \mathrm{w}, \mathrm{C}), \delta(\mathrm{w}, \mathrm{z}, \mathrm{C}), \delta(\mathrm{z}, \mathrm{w}, \mathrm{C})) \\
& =\gamma(\delta(\mathrm{z}, \mathrm{w}, \mathrm{C}))<\delta(\mathrm{z}, \mathrm{w}, \mathrm{C})
\end{aligned}
$$

we reach a contradiction. Thus, our supposition that the set $(\mathcal{F}[\mathrm{I}] \cap \mathcal{F}[\mathrm{J}] \cap \mathcal{F}[\mathrm{S}] \cap \mathcal{F}[\mathrm{T}] \cap \mathcal{F}[\mathrm{F}] \cap$ $\mathcal{F G}$ is not a singleton was wrong. Hence the set $\mathcal{F} \cap \mathcal{F} \mathrm{J} \cap \mathcal{F} \cap \cap \mathcal{F} \cap \cap \mathcal{F} F \mathcal{F} G$ is a singleton.

The following example illustrates Theorem 3.1.

Example: 3.1 Define $d$ on $[0,1] \times[0,1] \times[0,1]$ by $d(x, y, z)=\min \{\rho(x, y), \rho(y, z), \rho(z, x)\}$, where $\rho$ is a usual metric on $[0,1]$. Then it is easy to see that $d$ is a 2 -metric on $[0,1]$. Define $I, J:[0,1] \rightarrow[0,1]$ and $\mathrm{F}, \mathrm{G}:[0,1] \rightarrow \mathcal{B}([0,1])$ by

$$
\begin{array}{ll}
\mathrm{Ix}=\left\{\begin{array}{c}
\frac{1}{4}, \mathrm{x} \in\left[0, \frac{1}{4}\right], \\
\frac{\mathrm{x}+1}{8}, \mathrm{x} \in\left(\frac{1}{4}, 1\right],
\end{array}\right. & \mathrm{Jx}=\left\{\begin{array}{c}
1-3 \mathrm{x}, \mathrm{x} \in\left[0, \frac{1}{4}\right], \\
0, \mathrm{x} \in\left(\frac{1}{4}, 1\right],
\end{array}\right. \\
\mathrm{Fx}=\left\{\frac{1}{4}\right\}, \mathrm{x} \in[0,1], & \mathrm{Gx}=\left\{\begin{array}{c}
\left\{\frac{1}{4}\right\}, \mathrm{x} \in\left[0, \frac{1}{4}\right], \\
\left(\frac{5}{32}, \frac{1}{4}\right], \mathrm{x} \in\left(\frac{1}{4}, 1\right],
\end{array}\right.
\end{array}
$$

Let $\varphi:\left(\mathbb{R}^{+}\right)^{5} \rightarrow \mathbb{R}^{+}$be defined by $\varphi\left(t_{1}+t_{2}+t_{3}+t_{4}+t_{5}\right)=\frac{1}{7}\left(t_{1}+t_{2}+t_{3}+t_{4}+t_{5}\right)$. Obviously, the function $\varphi$ is continuous and increasing in each coordinate variable. Also $\gamma(\mathrm{t})=\varphi\left(\mathrm{t}, \mathrm{t}, \mathrm{a}_{1} \mathrm{t}, \mathrm{a}_{2} \mathrm{t}, \mathrm{t}\right)=\frac{\mathrm{t}}{7}\left[3+\left(\mathrm{a}_{1}+\mathrm{a}_{2}\right)\right]<t$, where $\mathrm{a}_{1}+\mathrm{a}_{2}=3$. Thus, the function $\varphi \in$ Ф.Notice that $\mathrm{UF}(\mathrm{X})=\left\{\frac{1}{4}\right\} \subseteq\{0\} \cup\left[\frac{1}{4}, 1\right]=\mathrm{J}(\mathrm{X})$ and $\mathrm{G}(\mathrm{X})=\left(\frac{5}{32}, \frac{1}{4}\right]=\mathrm{I}(\mathrm{X})$. Also $\mathrm{I}(\mathrm{X})$ and $\mathrm{J}(\mathrm{X})$ are complete subspaces of $X$.

Now, we consider the following cases:

Case: 1 If $x \in\left[0, \frac{1}{4}\right]$ and $y \in\left[0, \frac{1}{4}\right]$, then for $C \in \mathcal{B}([0,1])$, we have;

$$
\begin{aligned}
\delta(\mathrm{Fx}, \mathrm{Gy}, \mathrm{C}) & =\delta\left(\left\{\frac{1}{4}\right\},\left\{\frac{1}{4}\right\}, \mathrm{C}\right)=0 \\
& \leq \frac{1}{7}[\delta(\mathrm{Ix}, \mathrm{Jy}, \mathrm{C})+\delta(\mathrm{Ix}, \mathrm{Fx}, \mathrm{C})+\delta(\mathrm{Jy}, \mathrm{Gy}, \mathrm{C})+\mathcal{D}(\mathrm{Ix}, \mathrm{Gy}, \mathrm{C})+\mathcal{D}(\mathrm{Jy}, \mathrm{Fx}, \mathrm{C}] \\
& =\varphi(\delta(\mathrm{Ix}, \mathrm{Jy}, \mathrm{C}), \delta(\mathrm{Ix}, \mathrm{Fx}, \mathrm{C}), \delta(\mathrm{Jy}, \mathrm{Gy}, \mathrm{C}), \mathcal{D}(\mathrm{Ix}, \mathrm{Gy}, \mathrm{C}), \mathcal{D}(\mathrm{Jy}, \mathrm{Fx}, \mathrm{C}))
\end{aligned}
$$

Case: 2 If $x \in\left[0, \frac{1}{4}\right], y \in\left(\frac{1}{4}, 1\right]$ and $C=\{1\} \in \mathcal{B}([0,1])$, we have;

$$
\begin{aligned}
\delta(\mathrm{Fx}, \mathrm{Gy}, \mathrm{C}) & =\delta\left(\left\{\frac{1}{4}\right\},\left(\frac{5}{32}, \frac{1}{4}\right],\{1\}\right) \\
& =\sup \left\{\mathrm{d}(\mathrm{a}, \mathrm{b}, \mathrm{c}): \mathrm{a} \in\left\{\frac{1}{4}\right\}, \mathrm{b} \in\left(\frac{5}{32}, \frac{1}{4}\right], \mathrm{c} \in\{1\}\right\} \\
& \leq \frac{3}{32}=\frac{1}{7} \cdot \frac{21}{32}=\frac{1}{7}\left[\frac{1}{4}+0+\frac{5}{32}+0+\frac{1}{4}\right] \\
& =\frac{1}{7}\left[\delta\left(\frac{1}{4}, 0,\{1\}\right)+\delta\left(\frac{1}{4},\left\{\frac{1}{4}\right\},\{1\}\right)+\delta\left(0,\left(\frac{5}{32}, \frac{1}{4}\right],\{1\}\right)\right. \\
& \left.+\mathcal{D}\left(\frac{1}{4},\left(\frac{5}{32}, \frac{1}{4}\right],\{1\}\right)+\mathcal{D}\left(0,\left\{\frac{1}{4}\right\},\{1\}\right)\right]
\end{aligned}
$$


Point Theorems for Non-compatible, Discontinuous Hybrid Pairs of Mappings on 2-Metric Spaces by Using Implicit Relation

$$
\begin{aligned}
& =\frac{1}{7}[\delta(\mathrm{Ix}, \mathrm{Jy}, \mathrm{C})+\delta(\mathrm{Ix}, \mathrm{Fx}, \mathrm{C})+\delta(\mathrm{Jy}, \mathrm{Gy}, \mathrm{C})+\mathcal{D}(\mathrm{Ix}, \mathrm{Gy}, \mathrm{C})+\mathcal{D}(\mathrm{Jy}, \mathrm{Fx}, \mathrm{C})] \\
& =\varphi(\delta(\mathrm{Ix}, \mathrm{Jy}, \mathrm{C}), \delta(\mathrm{Ix}, \mathrm{Fx}, \mathrm{C}), \delta(\mathrm{Jy}, \mathrm{Gy}, \mathrm{C}), \mathcal{D}(\mathrm{Ix}, \mathrm{Gy}, \mathrm{C}), \mathcal{D}(\mathrm{Jy}, \mathrm{Fx}, \mathrm{C}))
\end{aligned}
$$

Case: 3 If $x \in\left(\frac{1}{4}, 1\right], y \in\left[0, \frac{1}{4}\right]$ and $C=\{1\} \in \mathcal{B}([0,1])$, then

$$
\begin{aligned}
\delta(\mathrm{Fx}, \mathrm{Gy}, \mathrm{C})= & \delta\left(\left\{\frac{1}{4}\right\},\left\{\frac{1}{4}\right\},\{1\}\right)=0 \\
& \leq \frac{1}{7} \delta(\mathrm{Ix}, \mathrm{Jy}, \mathrm{C})+\delta(\mathrm{Ix}, \mathrm{Fx}, \mathrm{C})+\delta(\mathrm{Jy}, \mathrm{Gy}, \mathrm{C})+\mathcal{D}(\mathrm{Ix}, \mathrm{Gy}, \mathrm{C})+\mathcal{D}(\mathrm{Jy}, \mathrm{Fx}, \mathrm{C}] \\
& =\varphi(\delta(\mathrm{Ix}, \mathrm{Jy}, \mathrm{C}), \delta(\mathrm{Ix}, \mathrm{Fx}, \mathrm{C}), \delta(\mathrm{Jy}, \mathrm{Gy}, \mathrm{C}), \mathcal{D}(\mathrm{Ix}, \mathrm{Gy}, \mathrm{C}), \mathcal{D}(\mathrm{Jy}, \mathrm{Fx}, \mathrm{C}))
\end{aligned}
$$

Case: 4 If $x \in\left(\frac{1}{4}, 1\right], y \in\left(\frac{1}{4}, 1\right]$ and $C=\{1\} \in \mathcal{B}([0,1])$, then

$$
\begin{aligned}
\delta(\mathrm{Fx}, \mathrm{Gy}, \mathrm{C}) & =\delta\left(\left\{\frac{1}{4}\right\},\left(\frac{5}{32}, \frac{1}{4}\right],\{1\}\right) \\
& =\sup \left\{\mathrm{d}(\mathrm{a}, \mathrm{b}, \mathrm{c}): \mathrm{a} \in\left\{\frac{1}{4}\right\}, \mathrm{b} \in\left(\frac{5}{32}, \frac{1}{4}\right], c \in\{1\}\right\} \leq \frac{3}{32}
\end{aligned}
$$

If $x=\frac{6}{32}$, then

$$
\begin{gathered}
\varphi(\delta(\mathrm{Ix}, \mathrm{Jy}, \mathrm{C}), \delta(\mathrm{Ix}, \mathrm{Fx}, \mathrm{C}), \delta(\mathrm{Jy}, \mathrm{Gy}, \mathrm{C}), \mathcal{D}(\mathrm{Ix}, \mathrm{Gy}, \mathrm{C}), \mathcal{D}(\mathrm{Jy}, \mathrm{Fx}, \mathrm{C})) \\
=\frac{1}{7}\left[\delta\left(\frac{19}{128}, 0,\{1\}\right)+\delta\left(\frac{19}{128},\left\{\frac{1}{4}\right\},\{1\}\right)+\delta\left(0,\left(\frac{5}{32}, \frac{1}{4}\right],\{1\}\right)\right. \\
\left.+\mathcal{D}\left(\frac{19}{128},\left(\frac{5}{32}, \frac{1}{4}\right],\{1\}\right)+\mathcal{D}\left(0,\left\{\frac{1}{4}\right\},\{1\}\right)\right]=\frac{85}{896}
\end{gathered}
$$

If $x=1$, then

$$
\begin{gathered}
\varphi(\delta(\mathrm{Ix}, \mathrm{Jy}, \mathrm{C}), \delta(\mathrm{Ix}, \mathrm{Fx}, \mathrm{C}), \delta(\mathrm{Jy}, \mathrm{Gy}, \mathrm{C}), \mathcal{D}(\mathrm{Ix}, \mathrm{Gy}, \mathrm{C}), \mathcal{D}(\mathrm{Jy}, \mathrm{Fx}, \mathrm{C})) \\
=\frac{1}{7}\left[\delta\left(\frac{1}{4}, 0,\{1\}\right)+\delta\left(\frac{1}{4},\left\{\frac{1}{4}\right\},\{1\}\right)+\delta\left(0,\left(\frac{5}{32}, \frac{1}{4}\right],\{1\}\right)\right. \\
\left.+\mathcal{D}\left(\frac{1}{4},\left(\frac{5}{32}, \frac{1}{4}\right],\{1\}\right)+\mathcal{D}\left(0,\left\{\frac{1}{4}\right\},\{1\}\right)\right]=\frac{3}{32}
\end{gathered}
$$

Thus, we have;

$$
\delta(\mathrm{Fx}, \mathrm{Gy}, \mathrm{C}) \leq \varphi(\delta \mathrm{d}(\mathrm{Ix}, \mathrm{Jy}, \mathrm{C}), \delta(\mathrm{Ix}, \mathrm{Fx}, \mathrm{C}), \delta(\mathrm{Jy}, \mathrm{Gy}, \mathrm{C}), \mathrm{D}(\mathrm{Ix}, \mathrm{Gy}, \mathrm{C}), \mathrm{D}(\mathrm{Jy}, \mathrm{Fx}, \mathrm{C}))
$$

for all $\mathrm{x} \in\left(\frac{1}{4}, 1\right]$ and $\mathrm{y} \in\left(\frac{1}{4}, 1\right]$. Hence, the considered implicit contraction (3.1) is satisfied. Also, $\mathcal{C}[\mathrm{I}, \mathrm{F}]=\left\{\frac{1}{4}, 1\right\} \neq \varnothing$ and $\mathcal{C}[\mathrm{I}, \mathrm{F}]=\left\{\frac{1}{4}\right\} \neq \varnothing$. If we take $\mathrm{x}=\frac{1}{4}$, then IIx $=\frac{1}{4}$ and FIx $=\left\{\frac{1}{4}\right\}$ and if we take $x=1$, then IIx $=\frac{1}{4}$ and FIx $=\left\{\frac{1}{4}\right\}$. Clearly, the pair $\{F, I\}$ is weakly commuting of type $(\mathrm{KB})$ for $\mathrm{x}=\frac{1}{4}$ and $\mathrm{x}=1$. Also if $\mathrm{x}=\frac{1}{4}$, then $J J x=\frac{1}{4}$ and $G J x=\left\{\frac{1}{4}\right\}$ and the pair $\{G, J\}$ is weakly commuting of type $(\mathrm{KB})$ for $\mathrm{x}=\frac{1}{4}$. Thus the pairs $\{\mathrm{F}, \mathrm{I}\}$ and $\{\mathrm{G}, J\}$ are weakly commuting of type $(\mathrm{KB})$ at coincidence points in X. Consequently all conditions of Theorem 3.1 are satisfied and hence, in view of Theorem 3.1, the set $(\mathcal{F}[\mathrm{I}] \cap \mathcal{F}[\mathrm{J}] \cap \mathcal{F}[\mathrm{F}] \cap \mathcal{F}[\mathrm{G}])$ is a singleton set. Obviously, $(\mathcal{F}[\mathrm{I}] \cap$ $\mathcal{F} \cap \mathcal{F F} \cap \mathcal{F G}=14$, that is, $14=\mathrm{I} 14=\mathrm{J} 14=\mathrm{F} 14=\mathrm{G} 14$.

Theorem: 3.1 Let (X, d) be a metric space. Let I, J be mappings of X into itself and F, G of X into $\mathcal{B}(X)$ satisfying the conditions (3.1) and (3.2). Suppose that I and F are D-maps and $U F(X)$ is closed or 


\section{R.D. Daheriya et al.}

J and $G$ are D-maps and $U G(X)$ is closed. Then $\mathcal{C}[\mathrm{I}, \mathrm{F}] \neq \varnothing$ and $\mathcal{C}[\mathrm{J}, \mathrm{G}] \neq \varnothing$. Further, if the hybrid pair $\{\mathrm{F}, \mathrm{I}\}$ and $\{\mathrm{G}, \mathrm{J}\}$ are weakly commuting of type $(\mathrm{KB})$ at coincidence points in $\mathrm{X}$, then $(\mathcal{F}[\mathrm{I}] \cap$ $\mathcal{F}[J] \cap \mathcal{F}[F] \cap \mathcal{F}[G])$ is a singleton set.

Proof Suppose that I and F are D-maps and $U F(X)$ is closed. Then there exists a sequence $\left\{x_{n}\right\}$ in $X$ such that $\lim _{n \rightarrow \infty} I_{n}=t$ and $\lim _{n \rightarrow \infty} F x_{n}=\{t\}$ for some $t \in X$. Since $U F(X)$ is closed and $\cup F(X) \subseteq$ $J(X)$, there is a point $u \in X$ such that $J u=t$.We will prove that $G u=\{t\}$. Suppose, to the contrary, that $\mathrm{Gu} \neq\{\mathrm{t}\}$. Since $\varphi$ is an increasing and continuous function, by (3.1), we obtain;

$$
\delta\left(\mathrm{Fx}_{\mathrm{n}}, \mathrm{Gu}, \mathrm{C}\right) \leq \varphi\left(\delta\left(\mathrm{Ix}_{\mathrm{n}}, \mathrm{Ju}, \mathrm{C}\right), \delta\left(\mathrm{Ix}_{\mathrm{n}}, \mathrm{Fx}_{\mathrm{n}}, \mathrm{C}\right), \delta(\mathrm{Ju}, \mathrm{Gu}, \mathrm{C}), \mathcal{D}\left(\mathrm{Ix}_{\mathrm{n}}, \mathrm{Gu}, \mathrm{C}\right), \mathcal{D}\left(\mathrm{Ju}, \mathrm{Fx} \mathrm{n}_{\mathrm{n}}, \mathrm{C}\right)\right)
$$

On letting $\mathrm{n} \rightarrow \infty$, we get;

$$
\begin{aligned}
\delta(\mathrm{t}, \mathrm{Gu}, \mathrm{C}) & \leq \varphi(\delta(\mathrm{t}, \mathrm{t}, \mathrm{C}), \delta(\mathrm{t}, \mathrm{t}, \mathrm{C}), \delta(\mathrm{t}, \mathrm{Gu}, \mathrm{C}), \delta(\mathrm{t}, \mathrm{Gu}, \mathrm{C}), \delta(\mathrm{t}, \mathrm{t}, \mathrm{C})) \\
& \leq \varphi(0,0, \delta(\mathrm{t}, \mathrm{Gu}, \mathrm{C}), \delta(\mathrm{t}, \mathrm{Gu}, \mathrm{C}), 0) \\
& \leq \varphi(\delta(\mathrm{t}, \mathrm{Gu}, \mathrm{C}), \delta(\mathrm{t}, \mathrm{Gu}, \mathrm{C}), 2 \delta(\mathrm{t}, \mathrm{Gu}, \mathrm{C}), \delta(\mathrm{t}, \mathrm{Gu}, \mathrm{C}), \delta(\mathrm{t}, \mathrm{Gu}, \mathrm{C})) \\
& =\gamma(\delta(\mathrm{t}, \mathrm{Gu}, \mathrm{C}))<\delta(\mathrm{t}, \mathrm{Gu}, \mathrm{C})
\end{aligned}
$$

we reach a contradiction. Thus, our supposition that $G u \neq t$ was wrong and hence $G u=\{t\}=\{J u\}$. This shows that $\mathcal{C}[J, G] \neq \varnothing$. Since the hybrid pair $\{\mathrm{G}, J\}$ is weakly commuting of type (KB) at coincidence points in $\mathrm{X}$, we have; $\delta(J J u, G J u, C) \leq R \delta(J u, G u, C)$ which gives $\{J J u\}=G J u$ or $\{J t\}=$ Gt. If JJu $\neq$ Ju, then by (3.1), we have;

$$
\delta\left(\mathrm{Fx}_{\mathrm{n}}, \mathrm{GJu}, \mathrm{C}\right) \leq \varphi\left(\delta\left(\mathrm{Ix}_{\mathrm{n}}, \mathrm{JJu}, \mathrm{C}\right), \delta\left(\mathrm{Ix}_{\mathrm{n}}, \mathrm{Fx}_{\mathrm{n}}, \mathrm{C}\right), \delta(J J u, \mathrm{GJu}, \mathrm{C}), \mathcal{D}\left(\mathrm{Ix}_{\mathrm{n}}, \mathrm{GJu}, \mathrm{C}\right), \mathcal{D}\left(J J u, \mathrm{Fx}_{\mathrm{n}}, \mathrm{C}\right)\right)
$$

On letting $n \rightarrow \infty$ and due to increasing property and continuity of $\varphi$, we get;

$$
\begin{aligned}
\delta(\mathrm{t}, \mathrm{GJu}, \mathrm{C}) & \leq \varphi(\delta(\mathrm{t}, \mathrm{JJu}, \mathrm{C}), \delta(\mathrm{t}, \mathrm{t}, \mathrm{C}), \delta(J J u, G J u, C), \mathcal{D}(\mathrm{t}, \mathrm{GJu}, \mathrm{C}), \mathcal{D}(J J u, t, C)) \\
\Rightarrow \delta(J u, J J u, C) & \leq \varphi(\delta(J u, J J u, C), 0,0, \delta(J u, J J u, C), \delta(J J u, J u, C)) \\
& \leq \varphi(\delta(J u, J J u, C), \delta(J u, J J u, C), \delta(J u, J J u, C), 2 \delta(J u, J J u, C), \delta(J J u, J u, C)) \\
& =\gamma(\delta(J u, J J u, C))<\delta(J u, J J u, C)
\end{aligned}
$$

a contradiction. Hence JJu $=\mathrm{Ju}$ and so $\{J J u\}=\mathrm{GJu}=\{J u\}=\mathrm{t}$. Since $\mathrm{U} \mathrm{G}(\mathrm{X}) \subseteq \mathrm{I}(\mathrm{X})$, there exists an element $\mathrm{v} \in \mathrm{X}$ such that $\mathrm{Gu}=\{\mathrm{Iv}\}$.We will show that $\mathrm{Fv}=\{\mathrm{Iv}\}$. If not, then the condition (3.1) gives;

$$
\begin{aligned}
\delta(\mathrm{Fv}, \mathrm{Gu}, \mathrm{C}) & \leq \varphi(\delta(\mathrm{Iv}, \mathrm{Ju}, \mathrm{C}), \delta(\mathrm{Iv}, \mathrm{Fv}, \mathrm{C}), \delta(\mathrm{Ju}, \mathrm{Gu}, \mathrm{C}), \mathcal{D}(\mathrm{Iv}, \mathrm{Gu}, \mathrm{C}), \mathcal{D}(\mathrm{Ju}, \mathrm{Fv}, \mathrm{C})) \\
\Rightarrow \delta(\mathrm{Fv}, \mathrm{Iv}, \mathrm{C}) & \leq \varphi(0, \delta(\mathrm{Iv}, \mathrm{Fv}, \mathrm{C}), 0,0, \delta(\mathrm{Iv}, \mathrm{Fv}, \mathrm{C})) \\
& \leq \varphi(\delta(\mathrm{Fv}, \mathrm{Iv}, \mathrm{C}), \delta(\mathrm{Fv}, \mathrm{Iv}, \mathrm{C}), 2 \delta(\mathrm{Fv}, \mathrm{Iv}, \mathrm{C}), \delta(\mathrm{Fv}, \mathrm{Iv}, \mathrm{C}), \delta(\mathrm{Fv}, \mathrm{Iv}, \mathrm{C})) \\
& =\gamma(\delta(\mathrm{Fv}, \mathrm{Iv}, \mathrm{C}))<\delta(\mathrm{Fv}, \mathrm{Iv}, \mathrm{C})
\end{aligned}
$$

a contradiction. Hence $\mathrm{Fv}=\{\mathrm{Iv}\}$. This means that $\mathcal{C}[\mathrm{I}, \mathrm{F}] \neq \varnothing$. Since the hybrid pair $\{\mathrm{F}, \mathrm{I}\}$ is weakly commuting of type (KB) at coincidence points in X, we have; $\delta(I I v, F J v, C) \leq \mathrm{R} \delta(I v, F v, C)$ which gives $\{$ IIv $\}=$ FIv or $\{I t\}=$ Ft. If IIv $\neq$ Iv, then by (3.1), we have;

$$
\begin{aligned}
& \delta(\text { FIv, Gu, C }) \leq \varphi(\delta(I I v, \text { Ju, C }), \delta(I I v, \text { FIv, C) } \delta(J u, G u, C), \mathcal{D}(I I v, \text { Gu, C }), \mathcal{D}(J u, \text { FIv, C })) \\
& \Rightarrow \delta(\operatorname{IIv}, \mathrm{Iv}, \mathrm{C}) \leq \varphi(\delta(\mathrm{IIv}, \mathrm{Iv}, \mathrm{C}), \delta(\mathrm{IIv}, \mathrm{IIv}, \mathrm{C}), \delta(\mathrm{Iv}, \mathrm{Iv}, \mathrm{C}), \delta(\mathrm{IIv}, \mathrm{Iv}, \mathrm{C}), \delta(\mathrm{Iv}, \mathrm{IIv}, \mathrm{C})) \\
& =\varphi(\delta(\operatorname{IIv}, \mathrm{Iv}, \mathrm{C}), 0,0, \delta(\mathrm{IIv}, \mathrm{Iv}, \mathrm{C}), \delta(\mathrm{Iv}, \mathrm{IIv}, \mathrm{C}))
\end{aligned}
$$




$$
\begin{aligned}
& \leq \varphi(\delta(I I v, \text { Iv, C }), \delta(I I v, \text { Iv, C), } 2 \delta(I I v, \text { Iv, C }), \delta(I I v, \text { Iv, C }), \delta(I I v, \text { Iv, C })) \\
& =\gamma(\delta(\operatorname{IIv}, \text { Iv, C }))<\delta(I I v, \text { Iv, C })
\end{aligned}
$$

This is a contradiction. Thus, IIv $=\mathrm{Iv}$ and $\operatorname{so}\{\mathrm{IIv}\}=\mathrm{FIv}=\{\mathrm{Iv}\}=\{\mathrm{Ju}\}=\mathrm{Gu}=\{\mathrm{t}\}$. Hence $\{\mathrm{t}\}=$ $\{\mathrm{It}\}=\{\mathrm{Jt}\}=\mathrm{Ft}=\mathrm{Gt}$ and so $(\mathcal{F}[\mathrm{I}] \cap \mathcal{F}[\mathrm{J}] \cap \mathcal{F}[\mathrm{F}] \cap \mathcal{F}[\mathrm{G}]) \neq \varnothing$. In view of Proposition 3.1, the set $(\mathcal{F}[\mathrm{I}] \cap \mathcal{F}[\mathrm{J}] \cap \mathcal{F}[\mathrm{F}] \cap \mathcal{F}[\mathrm{G}])$ is a singleton set. If one assumes that $\mathrm{J}$ and $\mathrm{G}$ are D-maps and $\cup \mathrm{G}(\mathrm{X})$ is closed, then analogous arguments establish that $\mathcal{C}[\mathrm{I}, \mathrm{F}] \neq \varnothing, \mathcal{C}[\mathrm{J}, \mathrm{G}] \neq \varnothing$ and the set $(\mathcal{F}[\mathrm{I}] \cap \mathcal{F}[\mathrm{J}] \cap \mathcal{F}[\mathrm{F}] \cap \mathcal{F}[\mathrm{G}])$ is a singleton set. This finishes the proof.

Now, if we put $\mathrm{F}=\mathrm{G}$ and $\mathrm{I}=\mathrm{J}$ in Theorem 3.1, then we obtain the following Corollary.

Corollary: 3.5 Let $(\mathrm{X}, \mathrm{d})$ be a 2-metric space. Let $\mathrm{I}$ be a mappings of $\mathrm{X}$ into itself and $\mathrm{F}$ of $\mathrm{X}$ into $\mathcal{B}(\mathrm{X})$ satisfying the following conditions:

$$
\delta(\mathrm{Fx}, \mathrm{Fy}, \mathrm{C}) \leq \varphi(\delta(\mathrm{Ix}, \mathrm{Iy}, \mathrm{C}), \delta(\mathrm{Ix}, \mathrm{Fx}, \mathrm{C}), \delta(\mathrm{Iy}, \mathrm{Fy}, \mathrm{C}), \mathcal{D}(\mathrm{Ix}, \mathrm{Fy}, \mathrm{C}), \mathcal{D}(\mathrm{Iy}, \mathrm{Fy}, \mathrm{C}))
$$

for all $\mathrm{x}, \mathrm{y} \in \mathrm{X}$. where $\varphi \in \Phi$. Suppose that $\mathrm{UF}(\mathrm{X}) \subseteq \mathrm{I}(\mathrm{X})$. Suppose I and F are D-maps and $\cup \mathrm{F}(\mathrm{X})$ is closed. If the pair $\{\mathrm{F}, \mathrm{I}\}$ is weakly commuting of type (KB) at coincidence points in $\mathrm{X}$, then $(\mathcal{F}[\mathrm{I}] \cap \mathcal{F}[\mathrm{F}])$ is a singleton set.

If we put $\mathrm{I}=\mathrm{J}$ in Theorem 3.1, then we obtain the following Corollary.

Corollary: 3.6 Let $(\mathrm{X}, \mathrm{d})$ be a 2-metric space. Let $\mathrm{I}$ be a mapping of $\mathrm{X}$ into itself and $\mathrm{F}, \mathrm{G}$ of $\mathrm{X}$ into $\mathcal{B}(\mathrm{X})$ satisfying the following conditions:

$$
\delta(\mathrm{Fx}, \mathrm{Gy}, \mathrm{C}) \leq \varphi(\delta(\mathrm{Ix}, \mathrm{Iy}, \mathrm{C}), \delta(\mathrm{Ix}, \mathrm{Fx}, \mathrm{C}), \delta(\mathrm{Iy}, \mathrm{Gy}, \mathrm{C}), \mathcal{D}(\mathrm{Ix}, \mathrm{Gy}, \mathrm{C}), \mathcal{D}(\mathrm{Iy}, \mathrm{Fx}, \mathrm{C}))
$$

for all $\mathrm{x}, \mathrm{y} \in \mathrm{X}$. where $\varphi \in \Phi$. Suppose that $\mathrm{UF}(\mathrm{X}) \subseteq \mathrm{I}(\mathrm{X})$ and $\cup \mathrm{G}(\mathrm{X}) \subseteq \mathrm{I}(\mathrm{X})$. Suppose that $\mathrm{I}$ and $\mathrm{F}$ are D-maps and $U \mathrm{~F}(\mathrm{X})$ is closed or $\mathrm{I}$ and $\mathrm{G}$ are $\mathrm{D}$-maps and $\cup \mathrm{G}(\mathrm{X})$ is closed. Then $\mathcal{C}[\mathrm{I}, \mathrm{F}] \neq$ $\varnothing$ and $\mathcal{C}[\mathrm{I}, \mathrm{G}] \neq \varnothing$. Further, if the hybrid pair $\{\mathrm{F}, \mathrm{I}\}$ and $\{\mathrm{G}, \mathrm{I}\}$ are weakly commuting of type (KB) at coincidence points in $\mathrm{X}$, then $(\mathcal{F}[\mathrm{I}] \cap \mathcal{F}[\mathrm{F}] \cap \mathcal{F}[\mathrm{G}])$ is a singleton set.

If we put $F=G$ in Theorem 3.1, then we obtain the following Corollary.

Corollary 3.7 Let $(X, d)$ be a 2-metric space. Let I, J be a mappings of $X$ into itself and $\mathrm{F}$ of $\mathrm{X}$ into $\mathcal{B}(\mathrm{X})$ satisfying the following conditions:

$$
\delta(\mathrm{Fx}, \mathrm{Fy}, \mathrm{C}) \leq \varphi(\delta(\mathrm{Ix}, \mathrm{Jy}, \mathrm{C}), \delta(\mathrm{Ix}, \mathrm{Fx}, \mathrm{C}), \delta(\mathrm{Jy}, \mathrm{Fy}, \mathrm{C}), \mathcal{D}(\mathrm{Ix}, \mathrm{Fy}, \mathrm{C}), \mathcal{D}(\mathrm{Jy}, \mathrm{Fx}, \mathrm{C}))
$$

for all $\mathrm{x}, \mathrm{y} \in \mathrm{X}$. where $\varphi \in \Phi$. Suppose $\mathrm{UF}(\mathrm{X}) \subseteq \mathrm{I}(\mathrm{X}) \cap \mathrm{J}(\mathrm{X})$. Suppose that $\mathrm{I}$ and $\mathrm{F}$ are D-maps or J and $G$ are D-maps. Also $\cup F(X)$ is closed. Then $\mathcal{C}[I, F] \neq \varnothing$ and $\mathcal{C}[J, F] \neq \varnothing$. Further, if the hybrid pair $\{\mathrm{F}, \mathrm{I}\}$ and $\{\mathrm{F}, \mathrm{J}\}$ are weakly commuting of type $(\mathrm{KB})$ at coincidence points in $\mathrm{X}$, then $(\mathcal{F}[\mathrm{I}] \cap$ $\mathcal{F} \mathrm{J} \cap \mathcal{F F}$ is a singleton set.

\section{REFERENCES}

[1] M. E. Abd El-Monsef, H. M. Abu-Donia and Kh. Abd-Rabou, Common fixed point theorems of single and set-valued mappings on 2-metric spaces, Appl. Math. Inf. Sci. 2 (2007), 185-194.

[2] L. B.Ćiric, A. Razani, S. Radenovic and J. S. Ume, Common fixed point theorems for families weakly compatible maps, Comput. Math. Appl., in press.

[3] S. Gahler, 2-metrische Raume und ihre topologische structure, Math. Nacher. 26 (1963), 115148.

[4] S. Gahler, Linear 2-nonmierte Raume, Math. Nacher. 27 (1964), 1-43.

[5] S. Gahler, Uber die unformesior barkat 2-metriche Raume, Math. Nacher. 28 (1965), 235-244.

[6] K. Iseki Fixed Point Theorems in 2-Metric Spaces, Math. Seminar Notes XIX, 1975. 


\section{R.D. Daheriya et al.}

[7] G. Jungck, Commuting mappings and fixed points, Amer. Math. Monthly 83 (1976), 261-263.

[8] G. Jungck, Compatible maps and common fixed points, Internat. J. Math. Math. Sci. 9 (1986), 771-779.

[9] G. Jungck and B. E. Rhoades, Fixed point for set valued functions without continuity, Internat. Indian J. Pure Appl. Math. 29 (1998), 227-238.

[10] M. S. Khan, On fixed point theorems in 2-metric space, Publications de 'einstitut math'ematique 41 (1980), 107-112.

[11] P. P. Murthy, S. S. Chang, Y. J. Cho and B. K. Sharma, Compatible mappings of type(A) and common fixed point theorem, Kyungpook. Math. J. 32 (1992), 203-216.

[12] S. V. R. Naidu and J. R. Prasad, Fixed point theorem in 2-metric space "revisited", Indian J. Pure Apple. Math. Sci. 17 (1986), 974-993.

[13] R. K. Namdeo and S. Dubey, Coincidence and fixed points of non-expansive type mappings on 2-matric spaces, International Mathematical Forum 2 (2007), 803-811.

[14] H. K. Pathak, S. M. Kang and J. H. Baek, Weak compatible mappings of type (A) and common fixed points, Kyungpook. Math. J. 35 (1995), 345-359.

[15] A. Razani and M. Shirdaryazdi, A common fixed point theorem of compatible maps in Menger space, Chaos, Solitons and Fractals 32 (2007), 26-34.

[16] S. Sessa, On a weak commutativity condition of mapping in fixed point considerations, Pub. Inst. Math. 32 (1982), 149-153.

[17] S. L. Singh and G. Adiga Chandrashekar, A fixed point theorem in a 2-metric space and an application, J. of Natural and Physical Science 15 (2001), 55-64.

[18] A. G. White Jr, 2-Banach spaces, Math. Nachr. 42 (1969), 43-60.

[19] I. Kubiaczyk, B. Deshpande, Noncompatibility, discontinuity in consideration of common fixed point of set and single-valued maps, Southeast Asian Bull. Math. 32 (2008), 467-474.

[20] R. P. Pant, Common fixed points of noncommuting mappings, J. Math. Anal. Appl. 188 (1994), 436-440.

[21] R. P. Pant, Common fixed point theorems for contractive maps, J. Math. Anal. Appl. 226 (1998), 251-258.

[22] R. P. Pant, Common fixed points of Lipschitz type mapping pair, J. Math. Anal. Appl. 240 (1999), 280-283.

[23] R. P. Pant, Discontinuity and fixed points, J. Math. Anal. Appl. 240 (1999), 284-289.

[24] H. K. Pathak, Y. J. Cho, S. M. Kang, Remarks on R-weakly commuting mappings and common fixed point theorems, Bull. Korean Math. Soc. 34 (1997), 247-257.

[25] S. Sharma, B. Deshpande, Fixed point theorems for set and single valued maps without continuity and compatibility, Demonstratio Math. 40 (2007), 649-658.

[26] S. Sharma, B. Deshpande, R. Pathak, Common fixed point theorems for hybrid pairs of mappings with some weaker conditions, Fasc. Math. 39 (2008), 53-67.

[27] Khan M.D., A Study of Fixed Point Theorems Doctoral Thesis, Aligarh Muslim University, 1984.

[28] Shaban Sedghi, Nabi Shobe, Haiyun Zhou, A common fixed point theorem in D* - metric spaces, Hindawi Publishing Corporation, Fixed Point Theory and Applications, Vol. 2007, Article ID 27906, 13 pages, doi:10.1155/2007/27906.

[29] Djoudi A., Khemis R., Fixed point theorems for set and single valued maps without continuity, Demonstratio Math. 38(3) (2005), 739-751. 